\title{
Isomorphism Theorems for Cohomology Groups of Weakly 1-Complete Manifolds
}

\author{
By \\ Takeo OHSAWA*
}

\section{Table of Contents}

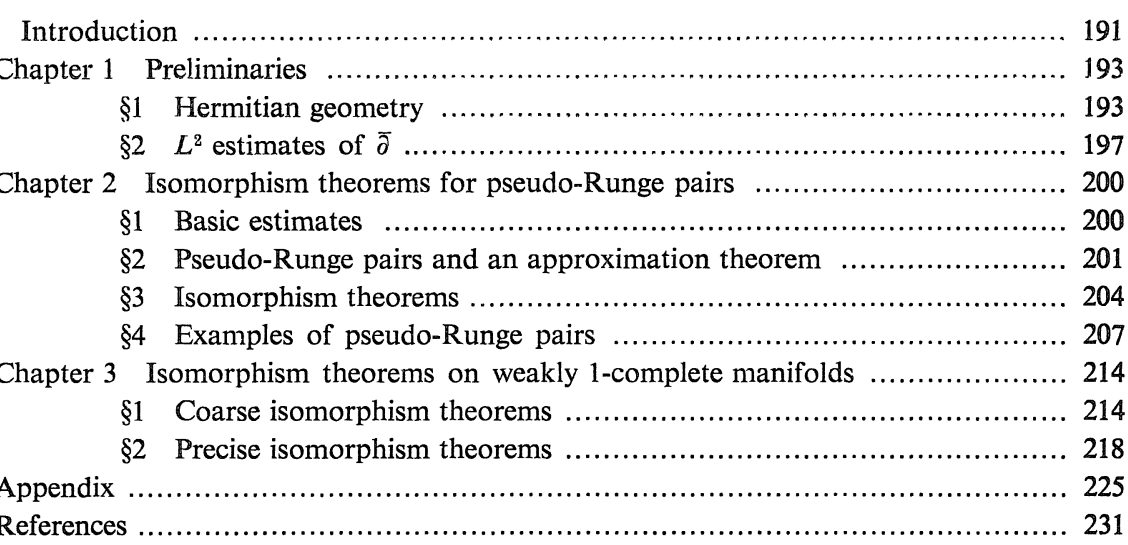

\section{Introduction}

In the theory of complex manifolds, there are two different extreme objects: compact manifolds and holomorphically complete ones. We have a lot of good knowledge about the fundamental properties of both classes of manifolds, contributions to which have been made by many celebrated authors in this century.

In 1970, S. Nakano [18] succeeded in solving a problem on the inverse of monoidal transformation by proving the vanishing of cohomology groups for line bundles over a class of complex manifolds. This class includes the above extremes and was called by him weakly 1-complete manifolds. The definition is as follows; a complex manifold is said to be weakly 1-complete if it carries a

Received February 9, 1981.

* Research Institute for Mathematical Sciences, Kyoto University, Kyoto 606, Japan. Supported by Alexander von Humboldt-Foundation. 
$C^{\infty}$ plurisubharmonic exhaustion function. It is trivial that a compact complex manifold is weakly 1-complete. It follows immediately from the Remmert's proper embedding theorem that holomorphically complete manifolds are weakly 1-complete.

From the definition, it is quite natural to expect that a weakly 1 -complete manifold is a nice intermediate object between compact complex manifolds and holomorphically complete ones.

In the last decade, more or less inspired by this philosophy, several authors have studied cohomological properties of weakly 1-complete manifolds: [1], $[12],[19],[20],[21],[22],[23],[24],[25],[26],[27],[28],[29]$. The following theorem is due to $\mathrm{S}$. Nakano [21].

Theorem 1. Let $X$ be a weakly 1-complete manifold and $\pi: B \rightarrow X$ a holomorphic line bundle. Assume that $B$ has a metric along the fibers whose curvature form is positive. Then,

$$
H^{q}\left(X, \Omega^{p}(B)\right)=0, \quad p+q>\operatorname{dim} X .
$$

Here we denote by $H^{q}\left(X, \Omega^{p}(B)\right)$ the q-th cohomology group of $X$ with coefficients in the sheaf of the germs of B-valued holomorphic p-forms.

Let $X_{c}$ be the sublevel set $\{x ; \varphi(x)<c\}$ of the exhaustion function $\varphi$. In [24], the author has extended the above theorem as follows:

Theorem 2. Let $X$ be a weakly 1-complete manifold and $\pi: B \rightarrow X \quad a$ holomorphic line bundle. Assume that $B$ has a metric along the fibers whose curvature form is positive outside a compact subset $K$ of $X$. Then, the natural restriction maps

$$
\rho_{c}: H^{q}\left(X, \Omega^{p}(B)\right) \longrightarrow H^{q}\left(X_{c}, \Omega^{p}(B)\right), \quad p+q>\operatorname{dim} X,
$$

are bijective if $X_{c} \supset K$.

The purpose of the present article is to extend the methods employed in [24] and show more explicitly how they unite each other to yield a fundamental theorem on cohomology groups of weakly 1-complete manifolds; the results including all the known ones will be deduced from the isomorphism theorem in Chapter 2.

As a by-product we obtain simple proofs of results obtained by AndreottiGrauert [3], Andreotti-Vesentini [4], and Hörmander [10]. Our viewpoint is that of [4] and the argument is essentially included in [10]; the use of complete 
metrics renders the derivation of the basic estimates very easy, and the argument borrowed from [10] enables us to avoid the use of so called 'bumping lemma'. Of course the advantage of our method lies in that we can prove the isomorphism theorems on weakly 1-complete manifolds in the same way.

The author expresses his hearty thanks to Professor S. Nakano who led him to this subject. $\mathrm{He}$ is also very grateful to Professor H. Grauert who allowed him to stay in Göttingen during the preparation of this paper and gave him kind advices. Last but not least he expresses many thanks to Mr. K. Takegoshi for careful reading of the manuscript and to the referee for valuable criticisms.

\section{Chapter 1. Preliminaries}

\section{§1. Hermitian Geometry}

Let $X$ be a paracompact complex manifold of dimension $n$ and $\pi: \mathrm{E} \rightarrow X$ a complex vector bundle. We denote by $E^{*}, \bar{E}, \stackrel{m}{\wedge} E$, and $E^{(m)}$, the dual, the conjugate, $m$-fold exterior power and $m$-fold symmetric power of $E$.

Definition 1.1. A section $h$ of $\operatorname{Hom}\left(E, \bar{E}^{*}\right)$ is called a hermitian metric along the fibers of $E$ if, for any point $x \in X$ and any two vectors $v, w \in E_{x}$ : $=\pi^{-1}(x)$,

$$
\left\{\begin{array}{l}
\overline{(h(x)(w))(\bar{v})}=(h(x)(v))(\bar{w}) \\
(h(x)(v))(\bar{v})>0, \quad v \neq 0
\end{array}\right.
$$

Hermitian metrics are assumed to be $C^{\infty}$ unless otherwise stated. Let $h$ be a hermitian metric along the fibers of $E$. For two sections $f$ and $g$ of $E$, we set

$$
\langle f, g\rangle=h(f)(\bar{g}) .
$$

$\langle f, g\rangle$ is called the pointwise inner product of $f$ and $g$. Canonically, $h$ induces metrics along the fibers of $E^{*}, \bar{E}, E^{(m)}, \stackrel{m}{\wedge} E$, and $\stackrel{p}{\wedge} E \otimes \stackrel{q}{\wedge} \bar{E}$. We also denote by $\langle$,$\rangle the pointwise inner product with respect to the induced metrics.$

Let $T_{X}$ be the tangent bundle of $X$ and $T_{X}^{\prime} \oplus T_{X}^{\prime \prime}$ the splitting of $T_{X}^{*} \otimes_{\mathbb{R}} \mathbb{C}$ into types $(1,0)$ and $(0,1)$ with respect to the complex structure of $T_{X}$. As a complex vector bundle we always identify $T_{X}$ (resp. $\left.T_{X}^{*}\right)$ with $T_{X}^{\prime *}\left(\operatorname{resp} . T_{X}^{\prime}\right)$.

Definition 1.2. A section of $E \otimes \stackrel{p}{\wedge} T_{X}^{\prime} \otimes \stackrel{q}{\wedge} T_{X}^{\prime \prime}$ is called an E-valued $(p, q)$-form. In particular, a section of $\stackrel{p}{\wedge} T_{X}^{\prime} \otimes \stackrel{q}{\wedge} T_{X}^{\prime \prime}$ is called a $(p, q)$-form. 
We can naturally identify $\stackrel{p}{\wedge}^{\prime} T_{X}^{\prime} \otimes \stackrel{q}{\wedge} T_{X}^{\prime \prime}$ with a subbundle of ${ }^{p+q}\left(T_{X} \otimes_{\mathbf{R}} \mathbf{C}\right)$. For simplicity we denote ${ }^{r}\left(T_{X} \otimes_{\mathbf{R}} \mathbb{C}\right)$ by $T_{X}^{r}$ and the subbundle of $T_{X}^{r}$ corresponding to $\stackrel{p}{\wedge}^{\prime} T_{X}^{\prime} \otimes \stackrel{q}{\wedge} T_{X}^{\prime \prime}$ by $T_{X}^{p, q}$. A section $u$ of $T_{X}^{r}$ is called an r-form. We set $\operatorname{deg} u=r$. We express $v \in\left(E \otimes T_{X}^{p, q}\right)_{x}$ as

$$
v=\sum_{\substack{i_{1}<\cdots<i_{p} \\ j_{1}<\cdots<j_{q}}} v_{i_{1} \cdots i_{p} \bar{J}_{1} \cdots \bar{J}_{q} q} \tau_{i_{1}} \wedge \cdots \wedge \tau_{i_{p}} \wedge \bar{\tau}_{j_{1}} \wedge \cdots \wedge \bar{\tau}_{j_{q}},
$$

where $v_{i_{1} \cdots i_{p} \bar{J}_{1} \cdots \bar{J}_{q}} \in E_{x}$ and $\left(\tau_{1}, \ldots, \tau_{n}\right)$ is a basis of $T_{X, x}^{\prime}$.

Let $d s^{2}$ be a hermitian metric along the fibers of $T_{X}$. We denote also by $\langle f, g\rangle$ the pointwise inner product of $E$-valued $(p, q)$-forms $f$ and $g$ with respect to the metric induced by $h$ and $d s^{2}$. The length of $f$ is defined by $|f|=\sqrt{\langle f, f\rangle}$. $d s^{2}$ is pointwise expressed as

$$
d s^{2}=\sum_{i=1}^{n} \tau_{i} \otimes \bar{\tau}_{i}
$$

for a suitable choice of the basis $\left(\tau_{1}, \ldots, \tau_{n}\right) . \quad\left(\tau_{1}, \ldots, \tau_{n}\right)$ is called an orthonormal basis with respect to $d s^{2}$. We set

$$
\omega=\sqrt{-1} \sum_{i=1}^{n} \tau_{i} \wedge \bar{\tau}_{i}
$$

$\omega$ is called the fundamental form associated to $d s^{2}$.

Let $E_{1}$ and $E_{2}$ be two complex vector bundles over $X$ provided with hermitian metrics along the fibers. Let $\xi: E_{1} \rightarrow E_{2}$ be a morphism or the conjugate of a morphism. The adjoint $\xi^{*}$ of $\xi$ is defined by the following formula:

$$
\langle\xi f, g\rangle_{2}=\left\langle f, \xi^{*} g\right\rangle_{1},
$$

where $f$ and $g$ run the sections of $E_{1}$ and $E_{2}$, respectively, and $\langle,\rangle_{i}$ denotes the pointwise inner product of $E_{i}$.

The conjugate star operator $\bar{*}_{E}: E \otimes T_{X}^{p, q} \rightarrow E^{*} \otimes T_{X}^{n-p, n-q}$ is defined to be a conjugate linear operator satisfying

$$
\left\{\begin{array}{l}
f \wedge \bar{*}_{E} f=\langle f, f\rangle d v \\
\left(\bar{*}_{E}\right)^{* *_{E}} f=(-1)^{p+q_{f}}
\end{array}\right.
$$

for any $E$-valued (p, q)-form $f$, where

$$
d v=\frac{1}{n !} \overbrace{\omega \wedge \cdots \wedge \omega}^{n}
$$

and 


$$
\begin{aligned}
& \left(e_{1} \otimes g_{1}\right) \wedge\left(e_{2}^{*} \otimes g_{2}\right)=e_{2}^{*}\left(e_{1}\right) g_{1} \wedge g_{2}, \\
& e_{1} \in E_{x}, e_{2}^{*} \in E_{X}^{*}, g_{1} \in T_{X, x}^{p, q}, g_{2} \in T_{X, x}^{p^{\prime}, q^{\prime}} .
\end{aligned}
$$

$d v$ is called the volume form with respect to $d s^{2} .{{ }^{*}}_{E}$ is unique. In particular, for the trivial bundle $\mathbb{1}$ with fiber $\mathbb{C}$ and with trivial metric along the fibers, we denote $\bar{*}_{1}$ by $\bar{*} . \quad \bar{*}$ operates naturally on $E \otimes T_{X}^{p, q}$ so that $\bar{*}(e \otimes f)=\bar{e} \otimes \bar{*} f$ for $e \otimes f \in\left(E \otimes T_{X}^{p, q}\right)_{x}$. We have $\bar{*}_{E}=\bar{h} \otimes \bar{*}$. Let $F$ be another complex vector bundle over $X$. For a $\operatorname{Hom}(E, F)$-valued $(p, q)$-form $f$, we denote by

$$
e(f): E \otimes T_{X}^{s, t} \longrightarrow F \otimes T_{X}^{s+p, t+q}
$$

the left multiplication by $f$. We set

$$
\left\{\begin{array}{l}
L=e(\omega) \\
\Lambda f=(-1)^{\operatorname{deg} f} \bar{*} * f, \text { for } f \in T_{X, x}^{r}
\end{array}\right.
$$

$L$ is called the Lefschetz' operator with respect to $d s^{2} . \quad \Lambda$ is the adjoint of $L$ with respect to the metrics induced by $d s^{2}$. We set $\bar{C} f=\sum(-1)^{q-p} \pi_{p, q} \bar{f}$, for $f \in T_{X}^{r}$, where we denote by $\pi_{p, q}$ the projection from $T_{X}^{p+q}$ to $T_{X}^{p, q}$. By abuse of notation we denote $\mathrm{id}_{E} \otimes L$ by $L$, and so on.

In what follows let $E$ be a holomorphic vector bundle. We denote by $C^{p, q}(X, E)$ the set of $E$-valued $(p, q)$-forms of class $C^{\infty}$. We set $C^{*}(X, E)$ $=\sum_{p, q} C^{p, q}(X, E)$. The complex exterior differentiations $\partial$ and $\bar{\partial}$ operate naturally on $C^{*}(X, \bar{E})$ and on $C^{*}(X, E)$, respectively. We set

$$
\begin{aligned}
& D_{E}=\bar{\partial}+h^{-1} \partial h \\
& D_{E}^{\prime}=h^{-1} \partial h \\
& \vartheta_{E}=-\bar{*}_{E} \bar{\partial} \bar{*}_{E}\left(=-\bar{*}^{-1} \bar{\partial} \bar{h} \bar{*}\right) \\
& \bar{\vartheta}=-\bar{*}_{\bar{*}} \bar{*}
\end{aligned}
$$

where by abuse of notation we denote $h \otimes$ id by $h$, and so on.

Theorem 1.3. Let the notations be as above. Then there is a linear operator $T_{1}$ (resp. $T_{2}$ ) belonging to the algebra of operators generated by $e(\bar{\partial} \omega)$ (resp. $e(\partial \omega)), L, \Lambda, \bar{*}$, and $\bar{C}^{-1}$ over the field of rational numbers, satisfying

$$
\begin{aligned}
& D_{E}^{\prime} \Lambda-\Lambda D_{E}^{\prime}=\sqrt{-1}\left(\vartheta_{E}+T_{1}\right) \\
& \left(\operatorname{resp} . \bar{\partial} \Lambda-\Lambda \bar{\partial}=-\sqrt{-1}\left(\bar{\vartheta}+T_{2}\right)\right)
\end{aligned}
$$


for any vector bundle $E$ and $h$. Furthermore $T_{i}$ are linear with respect to $d \omega$ and $T_{1}=T_{2}=0$ if $d \omega=0$.

Proof. See Appendix.

Theorem 1.4 (Extended Bochner-Calabi-Nakano formula).

$$
\begin{aligned}
- & \sqrt{-1}\left(D_{E}^{2} \Lambda-\Lambda D_{E}^{2}\right) \\
= & \bar{\partial} \vartheta_{E}+\vartheta_{E} \bar{\partial}-\left(\bar{\vartheta} D_{E}^{\prime}+D_{E}^{\prime} \bar{y}\right)+\bar{\partial} T_{1}+T_{1} \bar{\partial} \\
& -T_{2} D_{E}^{\prime}-D_{E}^{\prime} T_{2} .
\end{aligned}
$$

Proof. Since $\bar{\partial}^{2}=0$ and $\left(D_{E}^{\prime}\right)^{2}=0$,

$$
D_{E}^{2}=\left(\bar{\partial}+D_{E}^{\prime}\right)\left(\bar{\partial}+D_{E}^{\prime}\right)=\bar{\partial} D_{E}^{\prime}+D_{E}^{\prime} \bar{\partial} .
$$

Hence,

$$
\begin{aligned}
- & \sqrt{-1}\left(D_{E}^{2} \Lambda-\Lambda D_{E}^{2}\right) \\
= & -\sqrt{-1}\left(\bar{\partial} D_{E}^{\prime} \Lambda+D_{E}^{\prime} \bar{\partial} \Lambda-\Lambda \bar{\partial} D_{E}^{\prime}-\Lambda D_{E}^{\prime} \bar{\partial}\right) \\
= & -\sqrt{-1}\left(\bar{\partial}\left(D_{E}^{\prime} \Lambda-\Lambda D_{E}^{\prime}\right)+\left(D_{E}^{\prime} \Lambda-\Lambda D_{E}^{\prime}\right) \bar{\partial}\right. \\
& \left.+(\bar{\partial} \Lambda-\Lambda \bar{\partial}) D_{E}^{\prime}+D_{E}^{\prime}(\bar{\partial} \Lambda-\Lambda \bar{\partial})\right) \\
= & \bar{\partial} \vartheta_{E}+\vartheta_{E} \overline{\bar{\partial}}-\left(\bar{\vartheta} D_{E}^{\prime}+D_{E}^{\prime} \bar{\vartheta}\right)+\bar{\partial} T_{1}+T_{1} \bar{\partial} \\
& -T_{2} D_{E}^{\prime}-D_{E}^{\prime} T_{2} .
\end{aligned}
$$

We set

$$
(f, g)=\int_{X}\langle f, g\rangle d v
$$

for $f, g \in C^{p, q}(X, E)$ such that supp $f \cap \operatorname{supp} g \Subset X$, where supp $f$ denotes the support of $f$. Then, by Stokes' theorem,

$$
\begin{aligned}
& (\bar{\partial} f, g)=\left(f, \vartheta_{E} g\right), \\
& \left(D_{E}^{\prime} f, g\right)=(f, \bar{\vartheta} g),
\end{aligned}
$$

provided that supp $f \cap \operatorname{supp} g$ is compact. We put

$$
C_{0}^{p, q}(X, E)=\left\{f \in C^{p, q}(X, E) ; \operatorname{supp} f \text { is compact }\right\} .
$$

$C_{0}^{p, q}(X, E)$ is provided with the structure of a pre-Hilbert space with a norm $\|f\|=\sqrt{(f, f)}$. When we need to be more preciese, we denote $\|f\|$ by $\|f\|_{h, d s^{2}}$.

Combining Theorem 1.4 with arithmetic-geometric inequality we obtain

Theorem 1.5. Under the above notations, 


$$
\begin{aligned}
& \frac{3}{2}\left(\left\|\vartheta_{E} f\right\|^{2}+\|\bar{\partial} f\|^{2}\right) \\
& \geqq\left(-\sqrt{-1}\left(D_{E}^{2} \Lambda-\Lambda D_{E}^{2}\right) f, f\right) \\
& \quad-\frac{1}{2}\left(\left\|T_{1} f\right\|^{2}+\left\|T_{1}^{*} f\right\|^{2}+\left\|T_{2} f\right\|^{2}+\left\|T_{2}^{*} f\right\|^{2}\right), \quad f \in C_{0}^{p, q}(X, E) .
\end{aligned}
$$

Here $T_{i}^{*}$ denote the adjoints of $T_{i}$.

$D_{E}^{2}$ is a multiplication of a $\operatorname{Hom}(E, E)$-valued $(1,1)$-form. We set

$$
-D_{E}^{2}=e\left(\Theta_{h}\right), \quad \Theta_{h} \in C^{1,1}(X, \operatorname{Hom}(E, E)) .
$$

$\Theta_{h}$ is called the curvature form of $E$ with respect to $h$. Then (20) becomes

$$
\begin{gathered}
\frac{3}{2}\left(\left\|\vartheta_{E} f\right\|^{2}+\|\bar{\partial} f\|^{2}\right) \geqq \sqrt{-1}\left(\left(e\left(\Theta_{h}\right) \Lambda-\Lambda e\left(\Theta_{h}\right)\right) f, f\right) \\
-\frac{1}{2}\left(\left\|T_{1} f\right\|^{2}+\left\|T_{1}^{*} f\right\|^{2}+\left\|T_{2} f\right\|^{2}+\left\|T_{2}^{*} f\right\|^{2}\right) .
\end{gathered}
$$

$T_{i}$ and $T_{i}^{*}$ are called the torsions of $d s^{2}$. In what follows we call $d s^{2}$ a hermitian metric on $X$, and if $d \omega=0$ we say that $d s^{2}$ is (or simply $X$ is) Kählerian.

\section{§2. $\boldsymbol{L}^{2}$ Estimates of $\bar{\partial}$}

Let $H_{1}$ and $H_{2}$ be two Hilbert spaces with norms \|\|$_{i}, i=1,2$ and $T$ : $H_{1} \rightarrow H_{2}$ a linear operator with a domain of definition $D_{T} . \quad T$ is called a closed operator if $G_{T}:=\left\{(u, T u) ; u \in D_{T}\right\}$ is a closed subspace of $H_{1} \times H_{2}$. In what follows every linear operator is assumed to be closed and with a dense domain. $T^{*}: H_{2} \rightarrow H_{1}$, the adjoint of $T$, is defined as follows;

$$
G_{T^{*}}=\left\{(v, w) ;(u, w)_{1}=(T u, v)_{2} \quad \text { for any } u \in D_{T}\right\},
$$

where $(,)_{i}$ denote the inner products of $H_{i}$. We denote by $R_{T}\left(\operatorname{resp} . N_{T}\right)$ the range (resp. the kernel) of $T$. The closure of $R_{T}$ is denoted by $\left[R_{T}\right]$.

Assume that we are given another Hilbert space $H_{3}$ with a norm \|\|$_{3}$ and a linear operator $\mathrm{S}: \mathrm{H}_{2} \rightarrow \mathrm{H}_{3}$ such that

$$
S \circ T=0 .
$$

Then $N_{S} \supset R_{T}$ and $N_{T^{*}} \supset R_{S^{*} .}$ (2) implies that $R_{T}$ and $R_{S^{*}}$ are orthogonal, and the intersection of the orthogonal complements of these spaces is $\mathscr{H}:=N_{S}$ $\cap N_{T^{*}}$. Hence we have $H_{2}=\mathscr{H} \oplus\left[R_{T}\right] \oplus\left[R_{S^{*}}\right]$.

Theorem 2.1 (cf. Theorem 1.1.3 in [10]). Assume that from every sequence $g_{k} \in D_{T^{*}} \cap D_{S}$ with $\left\|g_{k}\right\|_{2}$ bounded and $T^{*} g_{k} \rightarrow 0$ in $H_{1}, S g_{k} \rightarrow 0$ in $H_{3}$, one can 
select a strongly convergent subsequence. Then both $R_{T}$ and $R_{S}$ are closed, and $\mathscr{H}$ is finite dimensional.

Elements of $\mathscr{H}$ are said to be harmonic.

Theorem 2.2 (cf. Theorem 1.1.4 in [10]). Let $F$ be a closed subspace of $\mathrm{H}_{2}$ containing $\mathrm{R}_{T}$. Assume that

$$
\|f\|_{2}^{2} \leqq\left\|T^{*} f\right\|_{1}^{2}+\|S f\|_{3}^{2}, \quad f \in D_{T^{*}} \cap D_{S} \cap F,
$$

then we have

$$
\text { If } g \in N_{S} \cap F \text {, we can find } u \in D_{T} \text { so that } T u=g \text { and }\|u\|_{1} \leqq\|h\|_{2} \text {. }
$$$$
\text { If } v \in R_{T^{*}} \text {, we can choose } f \in D_{T^{*}} \text { so that } T^{*} f=v \text { and }\|f\|_{2} \leqq\|v\|_{1} \text {. }
$$

Let the notations be as in Section 1 . We denote by $L^{p, q}(X, E)$ the space of square integrable $E$-valued $(p, q)$-forms on $X$ with respect to the metrics $h$ and $d s^{2}$. By Riesz-Fischer's theorem $L^{p, q}(X, E)$ is naturally identified with the completion of $C_{0}^{p, q}(X, E)$ with respect to the norm $\|f\|$. When we need to be more precise we denote $L^{p, q}(X, E)$ by $L^{p, q}\left(X, E, h, d s^{2}\right)$. $\bar{\partial}$ and other differential operators introduced in Section 1 are naturally extended to closed linear operators on $L^{p, q}(X, E)$ with dense domains

$$
\begin{aligned}
& D_{\bar{\partial}}=\left\{f \in L^{p, q}(X, E) ; \text { there is a } g \in L^{p, q+1}(X, E)\right. \text { such that } \\
& \left.\qquad\left(f, \vartheta_{E} \varphi\right)=(g, \varphi) \text { for any } \varphi \in C_{0}^{p, q+1}(X, E)\right\} .
\end{aligned}
$$

and so on. $\vartheta_{E}$, so extended, is called the formal adjoint of $\bar{\partial}$. When we need to be more precise we denote $D_{\bar{\partial}}, R_{\bar{\partial}}$, and $N_{\bar{\partial}}$ by $D_{\bar{\partial}}^{p, q}$ or $D_{\bar{\partial}}^{p}, q\left(h, d s^{2}\right)$, and so on.

In general $D_{\tilde{\partial}^{*}} \varsubsetneqq D_{\vartheta_{E}}$ because of the presence of the boundary of $X$. To avoid this technical difficulty we provide with $X$ a complete hermitian metric. This viewpoint is due to Andreotti-Vesentini [4].

Definition 2.3. We say a hermitian metric $d s^{2}$ is complete if the distance function on $X$ with respect to $d s^{2}$ provides $X$ with a structure of a complete metric space.

Theorem 2.4 (cf. Theorem 1.1 in [30] p. 22). If the hermitian metric $d s^{2}$ is complete, then

(6) $C_{0}^{p, q}(X, E)$ is dense in $D_{\bar{\partial}}^{p, q}$ with respect to the norm $\left(\|f\|^{2}+\|\bar{\partial} f\|^{2}\right)^{1 / 2}$.

(7) $C_{0}^{p, q}(X, E)$ is dense in $D_{\vartheta_{E}}^{p, q}$ with respect to the norm $\left(\|f\|^{2}+\left\|\vartheta_{E} f\right\|^{2}\right)^{1 / 2}$ 
(8) $C_{0}^{p, q}(X, E)$ is dense in $D_{\bar{\partial}}^{p, q} \cap D_{\vartheta_{E}}^{p, q}$ with respect to the norm

$$
\left(\|f\|^{2}+\|\bar{\partial} f\|^{2}+\left\|\vartheta_{E} f\right\|^{2}\right)^{1 / 2} .
$$

Corollary 2.5. If the hermitian metric $d s^{2}$ is complete, then $\bar{\partial}^{*}=\vartheta_{E}$ and $D_{E}^{\prime *}=\bar{\vartheta}$.

Proof. Clearly $D_{\bar{o}^{*}} \subset D_{\vartheta_{E}}$. Let $f \in D_{\vartheta_{E}}$. Then, by Theorem 2.4, there is a sequence $f_{k}$ in $C_{0}^{p, q}(X, E)$ such that $\left\|f_{k}-f\right\|+\left\|\vartheta_{E} f_{k}-\vartheta_{E} f\right\| \rightarrow 0$. Hence, for any $\varphi \in D_{\bar{\partial}}$, we have

$$
\left(\vartheta_{E} f, \varphi\right)=\lim _{k \rightarrow \infty}\left(\vartheta_{E} f_{k}, \varphi\right)=\lim _{k \rightarrow \infty}\left(f_{k}, \bar{\partial} \varphi\right)=(f, \bar{\partial} \varphi) .
$$

Therefore, $f \in D_{\bar{\partial}^{*}}$ and $\vartheta_{E} f=\bar{\partial}^{*} f$. The proof of $D_{E}^{\prime *}=\overline{0}$ is similar.

q.e.d.

Let

$$
\varphi=\varphi_{i_{1} \cdots i_{p}} \tau_{1} \wedge \cdots \wedge \tau_{n} \wedge \bar{\tau}_{i_{1}} \wedge \cdots \wedge \bar{\tau}_{i_{p}}
$$

where $\left(\tau_{1}, \ldots, \tau_{n}\right)$ is an orthonormal basis of $T_{X, x}^{\prime}$ with respect to $d s^{2}$. Then we have

$$
\left\langle\sqrt{-1} e\left(\tau_{i} \wedge \bar{\tau}_{i}\right) \Lambda \varphi, \varphi\right\rangle=0 \quad \text { if } \quad i \notin\left\{i_{1}, \ldots, i_{p}\right\}
$$

and

$$
\left\langle\sqrt{-1} e\left(\tau_{i_{\alpha}} \wedge \bar{\tau}_{i_{\alpha}}\right) \Lambda \varphi, \varphi\right\rangle=\langle\varphi, \varphi\rangle \quad \text { for } \quad 1 \leqq \alpha \leqq p .
$$

Hence, from (22) in Section 1, we obtain

Proposition 2.6. Suppose that the sums of $q$ eigenvalues of $\partial \bar{\partial} \Phi$ with respect to $d s^{2}$ are bigger than a nonnegative function $\lambda$. Then,

$$
\begin{aligned}
& \frac{3}{2}\left(\left\|\bar{\partial}^{*} f\right\|^{2}+\|\bar{\partial} f\|^{2}\right) \geqq \sqrt{-1}\left(e\left(\Theta_{h}\right) \Lambda f, f\right)+(\lambda f, f) \\
& \quad-\frac{1}{2}\left(\left\|T_{1} f\right\|^{2}+\left\|T_{1}^{*} f\right\|^{2}+\left\|T_{2} f\right\|^{2}+\left\|T_{2}^{*} f\right\|^{2}\right), \quad f \in C_{0}^{n, p}(X, E), p \geqq q .
\end{aligned}
$$

Similarly we have

Proposition 2.7. Suppose that the sums of $q$ eigenvalues of $\partial \bar{\partial} \Phi$ with respect to $d s^{2}$ are less than a nonpositive function $\lambda$. Then,

$$
\begin{array}{r}
\frac{3}{2}\left(\left\|\bar{\partial}^{*} f\right\|^{2}+\|\bar{\partial} f\|^{2}\right) \geqq-\sqrt{-1}\left(\Lambda e\left(\Theta_{h}\right) f, f\right)-(\lambda f, f) \\
-\frac{1}{2}\left(\left\|T_{1} f\right\|^{2}+\left\|T_{1}^{*} f\right\|^{2}+\left\|T_{2} f\right\|^{2}+\left\|T_{2}^{*} f\right\|^{2}\right), \\
f \in C_{0}^{0}, p(X, E), p \leqq n-q .
\end{array}
$$


In view of Theorem 2.2 and Theorem 2.4, the meaning of these propositions will be clear.

\section{Chapter 2. Isomorphism Theorems for Pseudo-Runge Pairs}

\section{§1. Basic Estimates}

Let $X$ be an $n$-dimensional complex manifold with a complete hermitian metric $d s^{2}$, and let $E$ be a holomorphic vector bundle over $X$ with a hermitian metric $h$ along the fibers.

Definition 1.1. We say that the basic estimate holds at bi-degree $(p, q)$ if there exist a compact subset $K \subset X$ and a constant $C_{0}$, satisfying

$$
\|f\|^{2} \leqq C_{0}\left\{\left\|\bar{\partial}^{*} f\right\|^{2}+\|\bar{\partial} f\|^{2}+\int_{K}\langle f, f\rangle d v\right\}, \quad f \in D_{\bar{\partial}^{*}}^{p, q} \cap D_{\bar{\partial}}^{p, q} .
$$

$K$ is called an exceptional set and $C_{0}$ is called a subellipticity constant.

Proposition 1.2. Assume that the basic estimate holds at bi-degree $(p, q)$. Then both $R_{\tilde{\partial}^{p}}^{p, q}$ and $R_{\tilde{\partial}^{*}}^{p, q-1}$ are closed, and $\operatorname{dim} N_{\vec{\partial}}^{p, q} / R_{\tilde{c}^{p}}^{p, q}<\infty$.

Proof. In view of Theorem 2.1 in Chapter 1 , we have only to show that from any sequence $g_{k} \in D_{\bar{\partial}^{*}}^{p, q} \cap D_{\bar{\partial}}^{p}, q$ with $\left\|g_{k}\right\|$ bounded and $\left\|\bar{\partial}^{*} g_{k}\right\| \rightarrow 0$ in $L^{p, q-1}(X, E),\left\|\bar{\partial} g_{k}\right\| \rightarrow 0$ in $L^{p, q+1}(X, E)$, one can select a strongly convergent subsequence.

By the completeness of the metric $d s^{2}$, we can take a sequence $f_{k} \in C_{0}^{p, q}(X, E)$ so that

$$
\begin{aligned}
& \left\|f_{k}-g_{k}\right\|<1 / k, \\
& \bar{\partial} f_{k} \rightarrow 0 \quad \text { in } \quad L^{p, q+1}(X, E) \text {, } \\
& \bar{\partial}^{*} f_{k} \rightarrow 0 \quad \text { in } \quad L^{p, q-1}(X, E)
\end{aligned}
$$

(cf. Theorem 2.4 in Chapter 1).

Let $K_{1}$ be any compact subset of $X$ and $K_{2}$ another compact subset of $X$ containing $K_{1}$ in the interior. Let $\chi: X \rightarrow \mathbf{R}$ be a $C^{\infty}$ function satisfying $\chi=1$ on $K_{1}$ and $\chi=0$ on $X-K_{2}$. Since

$$
\left(\left(\bar{\partial} \vartheta_{E}+\vartheta_{E} \bar{\partial}\right) f_{k}, f_{k}\right)+\left(f_{k}, f_{k}\right)
$$

is bounded,

$$
\left(\left(\bar{\partial} \vartheta_{E}+\vartheta_{E} \bar{\partial}\right)\left(\chi f_{k}\right), \chi f_{k}\right)+\left(\chi f_{k}, \chi f_{k}\right)
$$


is also bounded. Since $\bar{\partial} \vartheta_{E}+\vartheta_{E} \bar{\partial}$ is a strongly elliptic operator, in virtue of Gårding's inequality (cf. Theorem 6.5.1 in [16]) and Rellich's lemma (cf. Theorem 3.4.4 in [16]), $\chi f_{k}$ has a strongly convergent subsequence.

Hence $f_{h}$ has a subsequence $f_{i_{k}}$ converging strongly on $K$. By the basic estimate, $f_{i_{h}}$ converges strongly on $X$, hence so does $g_{i_{h}}$.

q.e.d.

\section{§2. Pseudo-Runge Pairs and an Approximation Theorem}

We shall present here an abstract form of Proposition 6 in [24] (cf. also Approximation theorem in [29]).

Let $X$ be a complex manifold and $E$ a holomorphic vector bundle over $X$. Let $X_{1}$ and $X_{2}$ be two open subsets of $X$.

Definition 2.1. The pair $\left(X_{1}, X_{2}\right)$ is called a pseudo-Runge pair at bi-degree $(p, q)$ with respect to $E$, if $X_{1} \subset X_{2}$ and there exist a complete hermitian metric $d s_{0}^{2}$ on $X_{1}$, a hermitian metric $h_{0}$ along the fibers of $\left.E\right|_{X_{1}}$, a sequence of complete hermitian metrics $d s_{k}^{2}(k=1,2, \ldots)$ on $X_{2}$, and a sequence of hermitian metrics $h_{k}$ along the fibers of $\left.E\right|_{X_{2}}$, satisfying the following properties;

(*) $d s_{k}^{2}, h_{k}$, and their derivatives converge on every compact subset of $X_{1}$ uniformly to $d s_{0}^{2}, h_{0}$, and to their derivatives, respectively.

(**) The basic estimates hold with respect to $d s_{k}^{2}$ and $h_{k}$ at bi-degree $(p, q+1)$ with a common subellipticity constant and a common exceptional set contained in $X_{1}$.

$(* * *) \quad L^{p, q}\left(X_{2}, E, h_{k}, d s_{k}^{2}\right) \subset L^{p, q}\left(X_{2}, E, h_{k+1}, d s_{h+1}^{2}\right)$ and there is a constant $C_{2}$ independent of $k$ such that

$$
\begin{aligned}
& \left\|\left.\varphi\right|_{X_{1}}\right\|_{h_{0}, d s_{0}^{2}} \leqq C_{2}\|\varphi\|_{h_{h}, d_{s}^{2}}, \\
& \varphi \in C_{0}^{p, q+i}\left(X_{2},\left.E\right|_{X_{2}}\right), \quad i=0,1 .
\end{aligned}
$$

For simplicity we set

$$
C_{0}^{p, q}\left(X_{1}, E\right)=C_{0}^{p, q}\left(X_{1},\left.E\right|_{X_{1}}\right)
$$

and so on.

Note that under the above conditions the basic estimate holds with respect to $h_{0}$ and $d s_{0}^{2}$, too.

The following lemma is essentially the same as Proposition 3.4 .5 in [10].

Lemma 2.2. Let $\left(X_{1}, X_{2}\right)$ be a pseudo-Runge pair at bi-degree $(p, q)$ with 
respect to $E$. Let $h_{0}, d s_{0}^{2}, h_{k}$, and $d s_{k}^{2}$ be chosen as above. Then there is an integer $k_{0}$ and a constant $C_{4}$ such that for any $k \geqq k_{0}$,

$$
C_{4}\left(\left\|\bar{\partial}^{*} f\right\|^{2}+\|\bar{\partial} f\|^{2}\right) \geqq\|f\|^{2},
$$

for any $f \in L^{p, q+1}\left(X_{2}, E, h_{k}, d s_{k}^{2}\right)$ satisfying $\left.f\right|_{X_{1}} \perp N_{\bar{c}} \cap N_{\bar{o}^{*}}$.

Proof. Assume that the assertion is false. Then there is a sequence $f_{k}$ satisfying

$$
\begin{aligned}
& f_{k} \in L^{p, q+1}\left(X_{2}, E, h_{k}, d s_{k}^{2}\right) \\
& \left\|f_{k}\right\|=1 \\
& \lim _{m \rightarrow \infty} \inf _{k \geqq m}\left\|\bar{\partial}^{*} f_{k}\right\|=0 \\
& \lim _{m \rightarrow \infty} \inf _{k \geqq m}\left\|\bar{\partial} f_{k}\right\|=0
\end{aligned}
$$

and

$$
\left.f_{k}\right|_{X_{1}} \perp N_{\bar{\partial}} \cap N_{\bar{\partial}^{*}}
$$

Choosing a subsequence if necessary, we may assume that

$$
\left\|\bar{\partial}^{*} f_{k}\right\|<\frac{1}{k}
$$

and

$$
\left\|\bar{\partial} f_{k}\right\|<\frac{1}{k} .
$$

Then there is a subsequence of $\left.f_{k}\right|_{X_{1}}$ weakly convergent in $L^{p, q+1}\left(X_{1}, E\right)$ and strongly convergent on a common exceptional set $K$ of the basic estimates. Let the weak limit be $f$. Then $f$ must be zero. In fact, by (7) we have $f \perp N_{\bar{\partial}} \cap N_{\tilde{o}^{*} \text {, }}$ and the completeness of the metric $d s_{0}^{2}$ implies that we have both $\bar{\partial} f=0$ and $\bar{\partial} * f=0$.

On the other hand, combining (8) and (9) with the basic estimates,

$$
\left.\int_{K}\left\langle f_{k}, f_{k}\right\rangle d v_{k}\right\rangle \frac{1}{C_{5}}-\frac{2}{k^{2}},
$$

where $C_{5}$ is a positive number and $d v_{k}$ denotes the volume form with respect to $d s_{k}^{2}$. From the strong convergence of $f_{k}$ on $K$, we obtain

$$
\int_{K}\langle f, f\rangle d v_{0} \geqq \frac{1}{C_{5}}
$$

where $d v_{0}$ denotes the volume form for $d s_{0}^{2}$. Therefore $f \neq 0$. A contradiction! 
Theorem 2.3 (Approximation theorem). Let $\left(X_{1}, X_{2}\right)$ be a pseudoRunge pair at bi-degree $(p, q)$ with respect to $E$. Let $h_{0}, d s_{0}^{2}, h_{k}$, and $d s_{k}^{2}$ be chosen as above, and let $f \in N_{\bar{\partial}}^{p}, q\left(h_{0}, d s_{0}^{2}\right)$. Then, for any positive number $\varepsilon$, there exist an integer $k_{0}$ and an $\tilde{f} \in N_{\bar{A}}^{p}, q\left(X_{2}, E, h_{k 0}, d s_{k_{0}^{2}}\right)$ satisfying

$$
\left\|\left.\tilde{f}\right|_{X_{1}}-f\right\|<\varepsilon \text {. }
$$

Proof. In virtue of Hahn-Banach's theorem, we have only to prove the following assertion:

Let $u \in L^{p, q}\left(X_{1}, E\right)$ and

$$
\left(u,\left.g\right|_{X_{1}}\right)=0, \quad g \in N_{\tilde{e}}^{p, q}\left(h_{k}, d s_{k}^{2}\right), \quad k=1,2, \ldots,
$$

then we have

$$
(u, f)=0, \quad f \in N_{\bar{\partial}}^{p, q}\left(h_{0}, d s_{0}^{2}\right) .
$$

To prove the assertion we observe first that by the assumption $(* * *)$,

$$
\left|\left(u,\left.v\right|_{X_{1}}\right)\right| \leqq C_{2}\|u\| \cdot\|v\| \text {. }
$$

Hence $\left(u,\left.\cdot\right|_{X_{1}}\right)$ is continuous on $L^{p, q}\left(X_{2}, E, h_{k}, d s_{k}^{2}\right)$ and its norm does not exceed $C_{2}\|u\|$. From the Riesz representation theorem there is a $u_{k} \in L^{p, q}\left(X_{2}\right.$, $\left.E, h_{k}, d s_{k}^{2}\right)$ such that

$$
\left\{\begin{array}{l}
\left(u_{k}, \cdot\right)=\left(u,\left.\cdot\right|_{X_{1}}\right) \\
\left\|u_{k}\right\| \leqq C_{2}\|u\| .
\end{array}\right.
$$

Clearly $u_{k}=0$ on $X_{2}-X_{1}$, so that $u_{k}$ are orthogonal to $N_{\bar{\partial}}^{p}, q\left(h_{k}, d s_{k}^{2}\right)$. Furthermore

$$
\left\|\left.u_{k}\right|_{X_{1}}\right\| \leqq C_{2}^{2}\|u\|
$$

In fact we have

$$
\left|\left(\left.u_{k}\right|_{X_{1}}, w\right)\right| \leqq C_{2}\left\|u_{k}\right\| \cdot\|w\| \leqq C_{2}^{2}\|u\| \cdot\|w\| .
$$

On the other hand, from $(*)$ combined with (16), we have

$$
\left(\left.u_{k}\right|_{X_{1}}, \varphi\right) \longrightarrow(u, \varphi),
$$

for any $\varphi \in C_{0}^{p, q}\left(X_{1}, E\right)$. (19), combined with (16), implies that

$$
\left(\left.u_{k}\right|_{X_{1}}, f\right) \longrightarrow(u, f), \quad \text { for any } f \in L^{p, q}\left(X_{1}, E\right) .
$$

Since $u_{k}$ are orthogonal to $N_{\bar{\delta}}^{p}, q\left(h_{k}, d s_{k}^{2}\right)$,

$$
u_{k} \in\left[R_{\widetilde{z}^{*}}^{p, q}\left(h_{k}, d s_{k}^{2}\right)\right] .
$$


Hence, in virtue of Lemma 2.2 and Theorem 2.2 in Chapter 1, there exist a constant $C_{3}$ and $w_{k} \in L^{p, q+1}\left(X_{2}, E, h_{k}, d s_{k}^{2}\right)$ satisfying

$$
\left\{\begin{array}{l}
\bar{\partial}^{*} w_{k}=u_{k} \\
\left\|w_{k}\right\| \leqq C_{3}\left\|u_{k}\right\| .
\end{array}\right.
$$

Since

$$
\left\|\left.w_{k}\right|_{X_{1}}\right\|\left(\leqq C_{2}\left\|w_{k}\right\| \leqq C_{2} C_{3}\left\|u_{k}\right\|\right) \leqq C_{2}^{2} C_{3}\|u\|,
$$

there is a subsequence of $\left.w_{k}\right|_{X_{1}}$ converging weakly in $L^{p, q+1}\left(X_{1}, E\right)$. Let the weak limit be $w$. Then

$$
(w, \bar{\partial} \varphi)=(u, \varphi), \quad \varphi \in C_{0}^{p, q}\left(X_{1}, E\right) .
$$

Therefore, in virtue of Corollary 2.5 in Chapter 1, we obtain

$$
\bar{\partial}^{*} w=u .
$$

Consequently,

$$
(u, f)=0, \quad \text { for any } f \in N_{\bar{i}}^{p, q}\left(h_{0}, d s_{0}^{2}\right) \text {. }
$$

q.e.d.

\section{§3. Isomorphism Theorems}

In this paragraph we shall prove isomorphism theorems for pseudo-Runge pairs. First we recall a fundamental fact about cohomology groups. Notations are as in Chapter 1.

Let $H^{q}\left(X, \Omega^{p}(E)\right)$ be the $q$-th cohomology group of $X$ with coefficients in the sheaf of holomorphic sections of $E \otimes \stackrel{p}{\wedge} T_{X}^{\prime}$. Let $L_{\text {loc }}^{p, q}(X, E)$ be the space of locally square integrable $E$-valued $(p, q)$-forms on $X . \quad L_{\text {loc }}^{p, q}(X, E)$ is naturally identified with the completion of $C^{p, q}(X, E)$ with respect to the semi-norms

$$
p_{k}(\psi)=\left(\int_{K}\langle\psi, \psi\rangle d v\right)^{1 / 2}, \quad \psi \in C^{p, q}(X, E)
$$

where $K$ runs through the compact subsets of $X$.

\section{Proposition 3.1.}

$$
\begin{aligned}
& H^{q}\left(X, \Omega^{p}(E)\right) \\
& \quad \cong\left\{f \in L_{\mathrm{loc}}^{p, q}(X, E) ; \bar{\partial} f=0\right\} /\left\{g \in L_{\mathrm{loc}}^{p, q}(X, E) ;\right. \text { there is an } \\
& \left.\quad h \in L_{\mathrm{loc}}^{p, q-1}(X, E) \text { satisfying } \bar{\partial} h=g\right\} .
\end{aligned}
$$

Here $\bar{\partial} u=v$ should read

$$
\left(u, \vartheta_{E} \varphi\right)=(v, \varphi) \text { for any } \varphi \in C_{0}^{p, q}(X, E), p \geqq 0, q \geqq 0 \text {. }
$$


Proof. Let $\mathscr{U}=\left\{U_{i}\right\}$ be a locally finite open cover of $X$ such that $U_{i}$ are biholomorphic to a polydisc. By solving the $\bar{\partial}$-equation for the domains of holomorphy in $\mathbf{C}^{n}$ (cf. [10] or [11]), we have

$$
\begin{aligned}
\{f \in & \left.L_{\mathrm{loc}}^{0, q}\left(U_{i_{1} \cdots i_{p}}, E\right) ; \bar{\partial} f=0\right\} \\
= & \left\{g \in L_{\mathrm{loc}}^{0, q}\left(U_{i_{1} \cdots i_{p}}, E\right) ; \text { there is an } h \in L_{\mathrm{loc}}^{0, q-1}\left(U_{i_{1} \cdots i_{p}}, E\right)\right. \\
& \text { satisfying } \bar{\partial} h=g\}, p \geqq 1, q \geqq 1 .
\end{aligned}
$$

Here we put $U_{i_{1} \cdots i_{p}}=U_{i_{1}} \cap \cdots \cap U_{i_{p}}$. It is easy to see that the equality (3) implies the equivalence (2).

Theorem 3.2 (Weak isomorphism theorem). Let $\left(X_{1}, X_{2}\right)$ be a pseudoRunge pair with respect to $E$, at bi-degrees $(p, q)$ and $(p, q+1)$. Then there is an integer $k_{0}$ such that the natural restriction maps

$$
\begin{aligned}
& \rho_{k}: N_{\overrightarrow{\hat{l}}}^{p, q+1}\left(h_{k}, d s_{k}^{2}\right) / R_{\overline{\vec{C}}}^{p, q+1}\left(h_{k}, d s_{k}^{2}\right) \longrightarrow \\
& N \overline{\bar{c}}_{\overline{\bar{c}}}^{p, q+1}\left(h_{0}, d s_{0}^{2}\right) / R_{\overline{\bar{c}}}^{p, q+1}\left(h_{0}, d s_{0}^{2}\right), \quad k \geqq k_{0}
\end{aligned}
$$

are bijective.

Proof. Since the basic estimate holds at bi-degree $(p, q+1)$, we have

$$
\operatorname{dim} N_{\vec{i}}^{p, q+1}\left(h_{0}, d s_{0}^{2}\right) / R_{\bar{i}}^{p}, q+1\left(h_{0}, d s_{0}^{2}\right)<\infty .
$$

Hence we have only to show that, for sufficiently large $k, \rho_{k}$ is injective and the image of $\rho_{k}$ is dense. The density of the images follows from Theorem 2.3. On the other hand, the injectivity follows at once from Lemma 2.2. q.e.d.

Given a pseudo-Runge pair $\left(X_{1}, X_{2}\right)$, we set for simplicity

$$
\left\{\begin{array}{l}
N^{p, q}\left(X_{1}\right)=N_{\overline{\bar{d}}}^{p, q}\left(h_{0}, d s_{0}^{2}\right) \\
R^{p, q}\left(X_{1}\right)=R_{\bar{c}}^{p, q}\left(h_{0}, d s_{0}^{2}\right)
\end{array}\right.
$$

Theorem 3.3 (Isomorphism theorem). Let $X$ be a complex manifold, let $E$ be a holomorphic vector bundle over $X$, and let $D$ be an open subset of $X$. Assume that there exist a family $X_{k}(k=1,2, \ldots)$ of open subsets of $X$ and $a$ family $D_{k}$ of open subsets of $D$ such that

$$
\begin{aligned}
& X=\bigcup_{k \geqq 1} X_{k}, \quad X_{k} \Subset X_{k+1} \\
& D=\bigcup_{k \geqq 1} D_{k}, \quad D_{k} \Subset D_{k+1} \\
& X_{1}=D_{1} \\
& X_{2}=D
\end{aligned}
$$


(11) $\left(X_{k}, X_{k+2}\right)$ is a pseudo-Runge pair at bi-degrees $(p, q)$ and $(p, q+1)$ with respect to $E$.

(12) $\left(D_{k}, D_{k+1}\right)$ is a pseudo-Runge pair at bi-degrees $(p, q)$ and $(p, q+1)$ with respect to $E$.

Then the natural restriction map

$$
\rho: H^{q+1}\left(X, \Omega^{p}(E)\right) \longrightarrow H^{q+1}\left(D, \Omega^{p}(E)\right)
$$

is bijective. Moreover, $H^{q+1}\left(X, \Omega^{p}(E)\right)$ and $H^{q+1}\left(D, \Omega^{p}(E)\right)$ are finite dimensional.

Proof. Injectivity: Let $f \in L_{\text {loc }}^{p, q+1}(X, E), \bar{\partial} f=0$, and $\left.f\right|_{D}=\bar{\partial} g$ for some $g \in L_{\mathrm{loc}}^{p, q}(D, E)$. By the pseudo-Rungeness of $\left(X_{1}, X_{3}\right)$ and Theorem 3.2, there is a $g_{1} \in L_{\text {loc }}^{p, q}\left(X_{3}, E\right)$ satisfying $\bar{\partial} g_{1}=\left.f\right|_{X_{3}}$. Thus, inductively we obtain $g_{k} \in L_{\mathrm{loc}}^{p, q}\left(X_{k+2}, E\right)$ satisfying $\bar{\partial} g_{k}=\left.f\right|_{X_{k+2}}$. By Theorem 2.3, there is a sequence $f_{k} \in L_{\text {loc }}^{p, q}\left(X_{k+3}, E\right)$ satisfying

$$
\left\{\begin{array}{l}
\bar{\partial} f_{k}=0 \\
\left\|\left.g_{k+1}\right|_{X_{k}}-\left.g_{k}\right|_{X_{k}}+\left.f_{k-1}\right|_{X_{k}}-\left.f_{k}\right|_{X_{k}}\right\|<\frac{1}{2^{k}},
\end{array}\right.
$$

where we put $f_{0}=0$, and the norm is taken with respect to a fixed metrics on $X$ and $E$. Hence we can define $\tilde{g} \in L_{\text {loc }}^{p, q}(X, E)$ by putting

$$
\tilde{g}=g_{k}-f_{k-1}+\sum_{m=1}^{\infty}\left(g_{k+m}-g_{k+m-1}+f_{k+m-2}-f_{k+m-1}\right)
$$

on $X_{k+2}$. Clearly, $\bar{\partial} \tilde{g}=f$.

Note that by the same argument we can prove that, in the following triangle of the natural restriction maps,

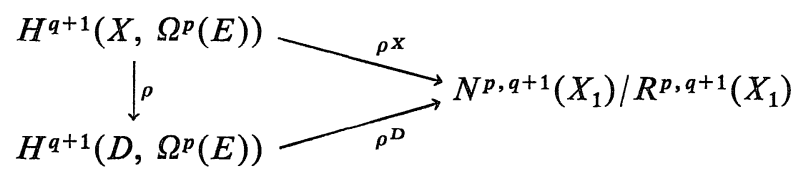

$\rho^{X}$ and $\rho^{D}$ are injective, too. Hence by Proposition $1.2 \operatorname{dim} H^{q+1}\left(X, \Omega^{p}(E)\right)$ $<\infty$ and $\operatorname{dim} H^{q+1}\left(D, \Omega^{p}(E)\right)<\infty$.

Surjectivity: Let $f \in L_{\mathrm{loc}}^{p, q+1}(D, E)$ and $\bar{\partial} f=0$. By Theorem 2.2, for any $\varepsilon>0$, there is an $f_{1} \in L_{\text {loc }}^{p, q+1}\left(X_{3}, E\right)$ satisfying

$$
\left\{\begin{array}{l}
\bar{\partial} f_{1}=0 \\
\left\|\left.f_{1}\right|_{X_{1}}-\left.f\right|_{X_{1}}\right\|<\frac{\varepsilon}{2}
\end{array}\right.
$$


Thus, inductively we can choose $f_{k} \in L_{\text {loc }}^{p, q+1}\left(X_{k+2}, E\right)$ satisfying

$$
\left\{\begin{array}{l}
\bar{\partial} f_{k}=0 \\
\left\|\left.f_{k}\right|_{X_{k}}-\left.f_{k-1}\right|_{X_{k}}\right\|<\frac{\varepsilon}{2^{k}},
\end{array}\right.
$$

where we put $f_{0}=f$. Hence we can define $\tilde{f} \in L_{\text {loc }}^{p, q+1}(X, E)$ by putting

$$
\tilde{f}=f_{k}+\sum_{m \geqq k}\left(f_{m+1}-f_{m}\right) \quad \text { on } \quad X_{k+2} \text {. }
$$

Clearly, $\tilde{f}$ satisfies

$$
\left\{\begin{array}{l}
\bar{\partial} \tilde{f}=0 \\
\left\|\left.\tilde{f}\right|_{X_{1}}-\left.f\right|_{X_{1}}\right\|<\sum_{k=1} \frac{\varepsilon}{2^{k}}=\varepsilon .
\end{array}\right.
$$

Therefore the image of $\rho^{X}$ is dense in the image of $\rho^{D}$. Combining this with the injectivity of $\rho^{D}$ and the finite dimensionality of $H^{q+1}\left(D, \Omega^{p}(E)\right)$, we obtain the surjectivity of $\rho$.

$$
\text { q.e.d. }
$$

\section{§4. Examples of Pseudo-Runge Pairs}

In accordance with Andreotti-Grauert [3], we adopt the following

Definition 4.1. Let $X$ be a complex manifold of dimension $n$, and let $q$ be a positive integer. $X$ is said to be strongly $q$-pseudoconvex (resp. strongly $q$-pseudoconcave) if there is a real-valued $C^{\infty}$ function $\Phi$ on $X$ satisfying

(1) $X_{c}:=\{x ; \Phi(x)<c\} \Subset X$ or $=X$, for any $c$,

(2) the Levi form of $\Phi$ has at least $n-q+1$ positive (resp. $n-q+1$ negative) eigenvalues outside a compact subset $K$ of $X$.

We call $\Phi$ an exhaustion function and $K$ an exceptional set.

Note that $X_{c}$ is also strongly $q$-pseudoconvex (resp. strongly $q$ pseudoconcave) if $X_{c} \supset K$.

Theorem 4.2. Let $X$ be a strongly q-pseudoconvex (resp. strongly $q$ pseudoconcave) manifold of dimension $n$ with an exhaustion function $\Phi$ and an exceptional set $K$, and let $E$ be a holomorphic vector bundle over $X$. Let $c<d, X_{c} \supset K$, and $X_{d} \Subset X$. Then, the pair $\left(X_{c}, X_{d}\right)$ is a pseudo-Runge pair with respect to $E$ at bi-degrees $(n, p), p \geqq q-1$ (resp. at bi-degrees $(0, p)$, $p \leqq n-q-2)$. 
For the proof we need the following lemmas.

Lemma 4.3 (cf. Lemma 4.1 in [30] § 12). Let $X$ be a paracompact complex manifold of dimension $n$, and let $\mathscr{L}$ be a real $C^{\infty}(1,1)$-form having at least $n-q+1$ positive eigenvalues. Then, for any positive integer $N$, there is a hermitian metric $d s^{2}$ on $X$ such that with respect to $d s^{2}$,

(3) at least $n-q+1$ eigenvalues of $\mathscr{L}$ are bigger than $N$ and

(4) negative eigenvalues of $\mathscr{L}$ are bigger than $-1 / N$.

Proof. By the continuity of the eigenvalues it is clear that there is a continuous hermitian metric on $X$ satisfying (3) and (4). Hence there is a $C^{\infty}$ hermitian metric satisfying (3) and (4).

q.e.d.

Lemma 4.4. There is a sequence $\lambda_{k}(t)(k=1,2, \ldots)$ of $C^{\infty}$ functions on $(-\infty, d)$, satisfying the following conditions.

$$
\begin{aligned}
& \lambda_{k}(t)=\frac{1}{\left(c+\frac{1}{k}-t\right)^{2}}+11 \text { on }(-\infty, c), \\
& \left\{\lambda_{k}^{\prime}(t)\right\}^{2}<11\left\{\lambda_{k}(t)\right\}^{3}, \\
& \int_{d-1}^{d} \sqrt{\lambda_{k}(t)} d t=\infty \\
& \lambda_{k}(t)>11, \lambda_{k}^{\prime}(t) \geqq 0 .
\end{aligned}
$$

Proof. We put

$$
\eta_{k}(t)=\left\{\begin{array}{l}
-\frac{1}{\left(c+\frac{1}{k}-t\right)^{2}}+11 \text { on }(-\infty, c] \\
2 k^{3}(t-c)+k^{2}+11 \text { on }\left(c, \frac{c+d}{2}\right] \\
\frac{(d-c)^{3} k^{3}}{8(d-t)^{2}}+\frac{(d-c) k^{3}}{2}+k^{2}+11 \text { on }\left(\frac{c+d}{2}, d\right) .
\end{array}\right.
$$

It is clear that $\eta_{k}(t)$ are differentiable and satisfy (5) to (8). Hence, approximating $\eta_{k}(t)$ by $C^{\infty}$ functions, we obtain a sequence $\lambda_{k}(t)$ of $C^{\infty}$ functions satisfying (5) to $(8)$.

\section{Lemma 4.5.}

$$
\begin{aligned}
(c-t)^{-m} e^{-\frac{1}{c-t}<2^{m}} m !\left(c+\frac{1}{k}-t\right)^{-m} \cdot e^{-\frac{1}{c+\frac{1}{k}-t}}, \\
k=1,2, \ldots, \quad m=0,1, \ldots, \quad t<c,
\end{aligned}
$$


where $e=\lim _{k \rightarrow \infty}\left(1+k^{-1}\right)^{k}$.

Proof. Left to the reader.

Proof of Theorem 4.2: Let $d s^{2}$ be a hermitian metric on $X$ and $h$ a hermitian metric along the fibers of $E$.

Pseudoconvex case: Let $X$ be strongly $q$-pseudoconvex. We may assume that outside $X_{c^{\prime}}$, such that $c^{\prime}<c$ and $X_{c^{\prime}} \supset K$, at least $\mathrm{n}-q+1$ eigenvalues of $\partial \bar{\partial} \Phi$ are bigger than $q$ and other eigenvalues are bigger than -1 . We put

$$
\left\{\begin{array}{l}
d s_{k}^{2}=\lambda_{k}(\Phi) d s^{2} \\
h_{k}=h e^{-A \int_{i n f \Phi}^{\Phi} \lambda_{k}(t) d t} .
\end{array}\right.
$$

Here $\lambda_{k}(t)$ is as in Lemma 4.4 , and $A$ is a positive number. By (7), $d s_{k}^{2}$ is a complete hermitian metric on $X_{d}$ (cf. Proposition 1 in [18] or Proposition 3.1 in [25]). Let $\omega_{k}$ be the fundamental form associated to $d s_{k}^{2}$ and let $\omega$ be associated to $d s^{2}$. Then

$$
d \omega_{k}=\lambda_{k}^{\prime}(\Phi) d \Phi \wedge \omega+\lambda_{k}(\Phi) d \omega .
$$

In virtue of $(6), e\left(d \omega_{k}\right)$ is a bounded operator with respect to the pointwise norm with respect to $d s_{k}^{2}$. Hence by Theorem 1.3 in Chapter 1 ,

$$
\begin{aligned}
& \left\{\begin{array}{l}
\left\|T_{i}^{k} \varphi\right\|_{h_{k}, d s_{k}^{2}} \leqq C_{6}\|\varphi\|_{h_{k}, d s_{k}^{2}} \\
\left\|T_{i}^{k *} \varphi\right\|_{h_{k}, d s_{k}^{2}} \leqq C_{6}\|\varphi\|_{h_{k}, d s_{k}^{2}}^{2}
\end{array}\right. \\
& i=1,2, \quad k=1,2, \ldots, \varphi \in C_{0}^{n, p}\left(X_{d}, E\right), p \geqq 0 \text {. }
\end{aligned}
$$

Here, $T_{i}^{k}$ and $T_{i}^{k *}$ are the torsions of $d s_{k}^{2}$, and $C_{6}$ is a constant which is independent of $A$. As for the curvature form of $E$ with respect to $h_{k}$, we have

$$
\Theta_{h_{k}}=\Theta_{h}+A\left\{\lambda_{k}(\Phi) \partial \bar{\partial} \Phi+\lambda_{k}^{\prime}(\Phi) \partial \Phi \wedge \bar{\partial} \Phi\right\} .
$$

Since the eigenvalues of $\lambda_{k}^{\prime}(\Phi) \partial \Phi \wedge \bar{\partial} \Phi$ are nonnegative and by the choice of $d s^{2}$ the sums of $q$ eigenvalues of $\lambda_{k}(\Phi) \partial \bar{\partial} \Phi$ with respect to $d s_{k}^{2}$ are bigger than 1 outside $X_{c^{\prime}}$, we deduce from Proposition 2.6 in Chapter 1 that

$$
\begin{aligned}
& 3\left\{\left\|\bar{\partial}^{*} \varphi\right\|^{2}+\|\bar{\partial} \varphi\|^{2}\right\} \\
& \geqq 2\left\{\sqrt{-1}\left(e\left(\Theta_{h}\right) \Lambda_{k} \varphi, \varphi\right)+A(\varphi, \varphi)\right\} \\
& \quad-\left\{\left\|T_{1}^{k} \varphi\right\|^{2}+\left\|T_{1}^{k *} \varphi\right\|^{2}+\left\|T_{2}^{k} \varphi\right\|^{2}+\left\|T_{2}^{k *} \varphi\right\|^{2}\right\}
\end{aligned}
$$

and whence, for sufficiently large $A$,

$$
\begin{aligned}
& \left\|\bar{\partial}^{*} \varphi\right\|^{2}+\|\bar{\partial} \varphi\|^{2} \\
& \quad \geqq\|\varphi\|^{2}, \text { for any } \varphi \in C_{0}^{n, p}\left(X_{d}-\overline{X_{c^{\prime}}}, E\right), p \geqq q .
\end{aligned}
$$


Here, the inner products and the norms as well as the torsions $T_{i}^{k}$ and the adjoints $\Lambda_{k}$ of the Lefschetz' operators are defined with respect to $d s_{k}^{2}$ and $h_{k}$. We fix such an $A$.

Let $\delta_{1}$ be a positive number satisfying $c^{\prime}<c^{\prime}+\delta_{1}<c-\delta_{1}<c$, and $\sigma$ a $C^{\infty}$ function on $X_{d}$ satisfying

$$
\sigma=\left\{\begin{array}{lll}
0 & \text { on } & X_{c^{\prime}+\delta_{1}} \\
1 & \text { on } & X_{c-\delta_{1}} .
\end{array}\right.
$$

Applying (16) to $\sigma \varphi$, where $\varphi \in C_{0}^{n, p}\left(X_{d}, E\right)$, we obtain the following estimate:

$$
\begin{gathered}
\left\|\bar{\partial}^{*} \varphi\right\|^{2}+\|\bar{\partial} \varphi\|^{2}+C_{7} \int_{X_{\mathrm{c}-\delta_{1}}}\langle\varphi, \varphi\rangle d v_{k} \\
\geqq\|\varphi\|^{2}, \quad \varphi \in C_{0}^{n, p}\left(X_{d}, E\right), \quad p \geqq q .
\end{gathered}
$$

Here $C_{7}$ is a constant and the norms are with respect to $h_{k}$ and $d s_{k}^{2}$.

Hence we obtain

$$
\begin{aligned}
& C_{8}\left\{\left\|\bar{\partial}^{*} f\right\|^{2}+\|\bar{\partial} f\|^{2}+\int_{X_{c-\delta_{1}}}\langle f, f\rangle d v_{k}\right\} \\
& \quad \geqq\|f\|^{2}, \quad f \in D_{\frac{\partial}{\partial}, p}^{n}\left(h_{k}, d s_{k}^{2}\right) \cap D_{\frac{\partial^{*}}{n}, p}\left(h_{k}, d s_{k}^{2}\right), \quad p \geqq q,
\end{aligned}
$$

for some constant $C_{8}$. Therefore the basic estimates hold for $d s_{k}^{2}$ and $h_{k}$ with common exceptional set $\overline{X_{c-\delta_{1}}} \subset X_{c}$ and common subellipticity constant $C_{8}$.

By the definition of $d s_{k}^{2}$ and $h_{k}$, they converge with their derivatives to hermitian metrics

$$
d s_{0}^{2}=\frac{d s^{2}}{(c-\Phi)^{2}}+11 d s^{2}
$$

and

$$
h_{0}=h \exp \left(-\frac{A}{c-\Phi}+\frac{A}{c-\inf \Phi}-11 A(\Phi-\inf \Phi)\right)
$$

respectively. The completeness of $d s_{0}^{2}$ is clear. So it remains to show that $(* * *)$ is true. That $L^{n, p}\left(X_{2}, E, h_{k}, d s_{k}^{2}\right) \subset L^{n, p}\left(X_{2}, E, h_{k+1}, d s_{k+1}^{2}\right)$ is clear. we note that

$$
\begin{aligned}
& \langle\varphi, \varphi\rangle_{h, d s_{0}^{2}} \leqq\langle\varphi, \varphi\rangle_{h, d s_{k}^{2}} \quad \text { on } \quad X_{c}, \\
& \varphi \in C^{s, t}\left(X_{d}, E\right), \quad s \geqq 0, t \geqq 0
\end{aligned}
$$

since 


$$
11+\frac{1}{(c-t)^{2}}>\lambda_{k}(t) \quad \text { on } \quad(-\infty, c) .
$$

Hence, by Lemma 4.5,

$$
\begin{aligned}
& \langle\varphi, \varphi\rangle_{h_{0}, d s_{0}^{2}} d v_{0} \leqq 2^{2 n}(2 n) !\langle\varphi, \varphi\rangle_{h_{k}, d s_{k}^{2}} d v_{k} \\
& \text { on } X_{c}, \quad \text { for } \varphi \in C^{s, t}\left(X_{d}, E\right), \quad s \geqq 0, t \geqq 0 .
\end{aligned}
$$

Thus $(*),(* *)$, and $(* * *)$ have been verified for the corresponding bi-degrees.

Pseudoconcave case: Assume that $X$ is strongly $q$-pseudoconcave. We set

$$
\left\{\begin{array}{l}
d s_{k}^{2}=d s^{2}+\lambda_{k}(\Phi) \partial \Phi \otimes \bar{\partial} \Phi \\
h_{k}=h \cdot e^{-B \int_{\text {inf } \Phi^{\lambda_{k}(t) d t}}^{\Phi},}
\end{array}\right.
$$

where $B$ is a positive number.

By (7), $d s_{k}^{2}$ are complete on $X_{d}$. Let $\omega_{k}$ and $\omega$ be as above. Then

$$
d \omega_{k}=d \omega+\lambda_{k}(\Phi) \bar{\partial} \partial \Phi \wedge(\partial \Phi+\bar{\partial} \Phi) .
$$

Hence the pointwise norms of $T_{i}^{k}$ and $T_{i}^{k *}, i=1,2$, with respect to $d s_{k}^{2}$, are bounded by $C_{10} \sqrt{\lambda_{k}(\Phi)}$ for some constant $C_{10}$. We may assume that outside some $X_{c^{\prime}}$ with $c^{\prime}<c$ and $X_{c^{\prime}} \supset K$, at least $n-q+1$ eigenvalues of $\partial \bar{\partial} \Phi$ with respect to $d s^{2}$ are less than $-q-3$ and other eigenvalues are less than 1 . Let $x \in X_{d}-X_{c^{\prime}}$ be any point, let $\gamma_{1} \geqq \cdots \geqq \gamma_{n}$ be the eigenvalues of $\partial \bar{\partial} \Phi$ at $x$ with respect to $d s^{2}$, and let $\gamma_{1}^{k} \geqq \cdots \geqq \gamma_{n}^{k}$ be the eigenvalues of $\partial \bar{\partial} \Phi$ with respect to $d s_{k}^{2}$ at $x$. Since the rank of $\lambda_{k}(\Phi) \partial \Phi \wedge \bar{\partial} \Phi$ is $\leqq 1$, by the minimum-maximum

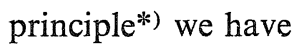

$$
\left\{\begin{array}{l}
\gamma_{n} \leqq \gamma_{n}^{k} \leqq \gamma_{n-1} \leqq \cdots \leqq \gamma_{q}^{k}<0 \\
\gamma_{i}^{k} \leqq \max \left\{\gamma_{1}, 0\right\}<1, \quad i<q
\end{array}\right.
$$

Hence

$$
\gamma_{n}^{k} \leqq \cdots \leqq \gamma_{q+1}^{k}<-q-3 .
$$

As for the curvature form, we have

$$
\Theta_{h_{k}}=\Theta_{h}+B\left(\lambda_{k}(\Phi) \partial \bar{\partial} \Phi+\lambda_{k}^{\prime}(\Phi) \partial \Phi \wedge \bar{\partial} \Phi\right) .
$$

By (6) and (8),

$$
\frac{\lambda_{k}^{\prime}(t)}{\lambda_{k}(t)}<\sqrt{11 \lambda_{k}(t)}<\lambda_{k}(t)
$$

*) The reader is referred to Courant-Hilbert's book 'Metoden der Mathematischen Physik I, Springer-Verlag, Berlin-Heidelberg-New York', Erstes Kapitel, Paragraph 4. 
Hence, if $\Gamma_{1} \geqq \cdots \geqq \Gamma_{n}$ are the eigenvalues of $\lambda_{k}(\Phi) \partial \bar{\partial} \Phi+\lambda_{k}^{\prime}(\Phi) \partial \Phi \wedge \bar{\partial} \Phi$ at $x$ with respect to $d s_{k}^{2}$, we have

$$
\left\{\begin{array}{l}
\Gamma_{n} \leqq \Gamma_{n-1} \leqq \cdots \leqq \Gamma_{q+1}<-(q+2) \lambda_{k}(\Phi(x)) \\
\Gamma_{q} \leqq \cdots \leqq \Gamma_{2}<\lambda_{k}(\Phi(x)) \\
\Gamma_{1}<2 \lambda_{k}(\Phi(x))
\end{array}\right.
$$

Therefore, for sufficiently large $B$, the sums of $q+1$ eigenvalues of $B\left(\lambda_{k}(\Phi) \partial \bar{\partial} \Phi\right.$ $\left.+\lambda_{k}^{\prime}(\Phi) \partial \Phi \wedge \bar{\partial} \Phi\right)$ are less than

$$
-B \lambda_{k}(\Phi(x)),
$$

at any point $x \in X_{d}-X_{c^{\prime}}$. Hence, for sufficiently large $B$, we deduce from Proposition 2.7 in Chapter 1 that, for any $\varphi \in C_{0}^{o, p}\left(X_{d}-\bar{X}_{c^{\prime}}, E\right)(P \leqq n-q-1)$,

$$
\begin{aligned}
& 3\left\{\left\|\vartheta_{k} \varphi\right\|_{k}^{2}+\|\bar{\partial} \varphi\|_{k}^{2}\right\} \\
& \quad \geqq 2\left\{-\sqrt{-1}\left(\Lambda_{k} e\left(\Theta_{h}\right) \varphi, \varphi\right)_{k}+B\left(\lambda_{k}(\Phi) \varphi, \varphi\right)_{k}\right\}-4 C_{10}^{2}\left(\lambda_{k}(\Phi) \varphi, \varphi\right)_{k} \\
& \quad \geqq B\left(\lambda_{k}(\Phi) \varphi, \varphi\right)_{k} \\
& \quad \geqq\|\varphi\|_{k}^{2} .
\end{aligned}
$$

Here we denote by $\vartheta_{k},\|\|_{k}, \Lambda_{k}$, and $(,)_{k}$ the formal adjoint of $\bar{\partial}$, the norm, the adjoint of the Lefschetz' operator, and the inner product with respect to $d s_{k}^{2}$ and $h_{k}$. Hence similarly as in pseudoconvex case, we obtain

$$
\begin{aligned}
& C_{11}\left(\left\|\bar{\partial}^{*} f\right\|^{2}+\|\bar{\partial} f\|^{2}+\int_{X_{c-\delta_{2}}}\langle f, f\rangle d v_{k}\right) \\
& \quad \geqq\|f\|^{2}, \quad f \in D_{\bar{\partial}}^{0}, p\left(h_{k}, d s_{k}^{2}\right) \cap D_{\bar{\partial}^{*}}^{0, p}\left(h_{k}, d s_{k}^{2}\right), \quad p \leqq n-q-1,
\end{aligned}
$$

with a common exceptional set $\overline{X_{c-\delta_{2}}}$ contained in $X_{c}$ and a common subellipticity constant $C_{11}$.

Now $d s_{k}^{2}$ and $h_{k}$ converge to

$$
d s_{0}^{2}=d s^{2}+\frac{\partial \Phi \otimes \bar{\partial} \Phi}{(c-\Phi)^{2}}+11 \partial \Phi \otimes \bar{\partial} \Phi
$$

and

$$
h_{0}=h \exp \left(-\frac{B}{c-\Phi}+\frac{B}{c-\inf \Phi}-11 B(\Phi-\inf \Phi)\right),
$$

respectively. Clearly $d s_{0}^{2}$ is complete. Thus (*) and (**) have verified. The verification of $(* * *)$ is the same as in the pseudoconvex case.

q.e.d.

Theorem 4.6. Let the situations be as in Theorem 4.2, and let $d s^{2}$ and $h$ be a hermitian metric on $X$ and a hermitian metric along the fibers of $E$, respectively. 
(32) If $X$ is strongly q-pseudoconvex and $X_{c} \supset K$, then the natural restriction maps

$$
\rho_{1}: H^{p}\left(X, \Omega^{n}(E)\right) \longrightarrow H^{p}\left(X_{c}, \Omega^{n}(E)\right), \quad p \geqq q,
$$

are bijective. Furthermore, if $p \geqq q, H^{p}\left(X, \Omega^{n}(E)\right)$ are finite dimensional and every cohomology class of $H^{p}\left(X_{c}, \Omega^{n}(E)\right)$ is represented uniquely by a harmonic form with respect to the following metrics:

$$
\left\{\begin{array}{l}
d s_{1}^{2}=\frac{d s^{2}}{(c-\Phi)^{2}} \\
h_{1}=h \cdot e^{-\frac{\alpha}{c-\Phi}}, \quad \alpha \gg 0 .
\end{array}\right.
$$

(33) If $X$ is strongly $q$-pseudoconcave and $X_{c} \supset K$, then the natural restriction maps

$$
\rho_{2}: H^{p}(X, E) \longrightarrow H^{p}\left(X_{c}, E\right), \quad p \leqq n-q-1
$$

are bijective. Furthermore, if $p \leqq n-q-1, H^{p}(X, E)$ are finite dimensional and every cohomology class of $H^{p}\left(X_{c}, E\right)$ is represented uniquely by a harmonic form with resepect to the following metrics:

$$
\left\{\begin{array}{l}
d s_{2}^{2}=d s^{2}+\frac{\partial \Phi \otimes \bar{\partial} \Phi}{(c-\Phi)^{2}} \\
h_{2}=h \cdot e^{-\frac{\alpha}{c-\Phi}}, \quad \alpha \gg 0 .
\end{array}\right.
$$

Proof. Combining Theorem 4.2 with Theorem 3.3, we obtain the former parts of (32) and (33). The latter parts follow from the proof of Theorem 4.2, since the two metrics $\lambda_{k}(\Phi) d s^{2}$ (resp. $d s^{2}+\lambda_{k}(\Phi) \partial \Phi \otimes \bar{\partial} \Phi$ ) and $\alpha \lambda_{k}(\Phi) d s^{2}$ (resp. $\left.d s^{2}+\alpha \lambda_{k}(\Phi) \partial \Phi \otimes \bar{\partial} \Phi\right)$ are equivalent if $\alpha>0$, so the closedness of the range of $\bar{\partial}$ is also valid for the above metrics.

q.e.d.

Letting $K=\varnothing$ and $c=\inf \Phi-1$ in the above theorem, we have

Corollary 4.7. Let $X$ be a strongly q-pseudoconvex manifold with empty exceptional set. Then,

$$
H^{p}\left(X, \Omega^{n}(E)\right)=0, \quad p \geqq q,
$$

for any holomorphic vector bundle $E$ over $X$. Moreover, if $X_{c} \Subset X$, then for any $f \in L^{n, p}\left(X_{c}, E, d s_{1}^{2}, h_{1}\right), p \geqq q$, with $\bar{\partial} f=0$, there is a $g \in L^{n, p-1}\left(X_{c}, E\right.$, $\left.d s_{1}^{2}, h_{1}\right)$ with $\bar{\partial} g=f$ and $\|g\| \leqq C\|f\|$, where $C$ is a constant independent of $f$.

A new feature of Theorem 4.6 is that the harmonic forms representing the cohomology classes need not satisfy any kind of boundary conditions, which 
was not the case in Hörmander's work [10]. In fact, in virtue of Stampacchia Inequality (cf. Theorem 1.2 in [30]), the completeness of the hermitian metric implies that $f$ is harmonic if and only if $\left(\bar{\partial} \vartheta_{E}+\vartheta_{E} \bar{\partial}\right) f=0$ in the sense described in (2), Section 3.

The advantage of using extended Bochner-Calabi-Nakano formula will be shown in the next chapter.

\section{Chapter 3. Isomorphism Theorems on Weakly 1-Complete Manifolds}

In this chapter we shall present several extensions and variations of so called 'vanishing theorems' on weakly 1-complete manifolds. Contrary to Theorem 4.6 in Chapter 2, the working hypothesis is the positivity of the curvature forms of the metrics along the fibers of holomorphic vector bundles. Two different notions of positivity are well known; one is due to S. Nakano [17] and the other is due to P. A. Griffiths [8], [9]. Both of these shall be examined here.

\section{§1. Coarse Isomorphism Theorems}

Let $X$ be a complex manifold of dimension $n$. For a vector space $V, S \triangleleft V$ shall mean that $S$ is a subspace of $V$.

Definition 1.1. A holomorphic vector bundle $E \rightarrow X$ with a hermitian metric $h$ along the fibers, in short a hermitian vector bundle $(E, h)$, is said to be $q$ positive (resp. q-negative) if, for any point $x \in X$, there is a subspace $S_{x} \triangleleft T_{X, x}$ of dimension $n-q+1$ such that $(h \otimes i d)\left(\left.\Theta_{h}\right|_{S_{X}}\right)$ is a positive definite (resp. negative definite) hermitian form on $E_{x} \otimes S_{x}$.

Note that if $E$ is a line bundle, i.e. if the rank of $E$ is 1 , then $(E, h)$ is qpositive (resp. q-negative) if and only if the curvature form $\Theta_{h}$ has everywhere at least $n-q+1$ positive eigenvalues (resp. negative eigenvalues). It follows from the definition of strongly q-pseudoconvex manifolds (resp. strongly qpseudoconcave manifolds) that every holomorphic vector bundle over them is q-positive (resp. q-negative) outside a compact subset.

The following definition is due to Nakano [19].

Definition 1.2. $X$ is said to be weakly 1-complete if there is a $C^{\infty}$ plurisubharmonic function $\Phi: X \rightarrow \mathbb{R}$ such that $X_{c}:=\{x ; \Phi(x)<c\} \Subset X$ for any $c$. $\Phi$ is called an exhaustion function. 
In what follows, let $X$ be a weakly 1-complete manifold of dimension $n$ with an exhaustion function $\Phi$ and a hermitian metric $d s^{2}$.

Theorem 1.3. Let $X$ be a weakly 1-complete manifold and let $(B, a)$ be a hermitian line bundle over $X$. Assume that $(B, a)$ is q-positive outside a compact subset $K \subset X_{c}$. Then, for any holomorphic vector bundle $E$ over $X$, there is an integer $m_{0}$ such that $H^{p}\left(X_{c}, E \otimes B^{m}\right), m>m_{0}$, are finite dimensional and the natural restriction maps

$$
H^{p}\left(X_{c}, E \otimes B^{m}\right) \longrightarrow H^{p}\left(X_{d}, E \otimes B^{m}\right), \quad m>m_{0}, c>d,
$$

are bijective, if $X_{d} \supset K$ and $p \geqq q$.

As a special case, we have

Corollary 1.4. Let the situations be as above. If $K=\emptyset$, then for any holomorphic vector bundle $E \rightarrow X$, there is an integer $m_{0}$ such that

$$
H^{p}\left(X_{c}, E \otimes B^{m}\right)=0, \quad \text { for } p \geqq q \text { and } m>m_{0} .
$$

For the proof we need the following

Lemma 1.5. Let $H_{1}$ and $H_{2}$ be two hermitian matrices of rank $n$, and let $\gamma_{1} \geqq \cdots \geqq \gamma_{n}$ be the eigenvalues of $H_{1}$. Assume that $\gamma_{1} \geqq \cdots \geqq \gamma_{n-q+1} \geqq \varepsilon>0$ and $\mathrm{H}_{2}$ is positive semi-definite. Let $v_{1}, \ldots, v_{n-q+1}$ be the eigenvectors of $H_{1}$ with $H_{1} v_{i}=\gamma_{i} v_{i}$, and set

$$
V=\left\{v \in \mathbb{C}^{n} ; v=\sum_{i=1}^{n-q+1} c_{i} v_{i}, c_{i} \in \mathbb{C}\right\}
$$

Then,

$$
\frac{{ }^{t} v H_{1} v+\varepsilon^{t} v H_{2} v}{\sum_{\alpha=1}^{n}\left|v^{\alpha}\right|^{2}+{ }^{t} v H_{2} v} \geqq \varepsilon, \quad \text { for } \quad v \in V-\{0\},
$$

where we put $v={ }^{t}\left(v^{1}, \ldots, v^{n}\right)$.

Proof. Trivial.

Proof of Theorem 1.3: We have to verify that for sufficiently large $m$, $\left(X_{d}, X_{c}\right)$ is a pseudo-Runge pair at bi-degrees $(n, p), p \geqq q-1$, with respect to $K_{X}^{*} \otimes E \otimes B^{m}$, where $K_{X}$ denotes the canonical bundle of $X$. Let $d s^{2}$ be so chosen that at any point $x \in X-X_{d-\delta}$ the eigenvalues $\gamma_{1} \geqq \cdots \geqq \gamma_{n}$ of $\Theta_{a}$ satisfy

$$
\left\{\begin{array}{l}
\gamma_{i}>q, \quad 1 \leqq i \leqq n-q+1 \\
\gamma_{i}>-1, \quad n-q+1<i \leqq n,
\end{array}\right.
$$


where $\delta$ is fixed so that $K \subset X_{d-\delta} \subset X_{d}$, and let $h$ be a hermitian metric along the fibers of $K_{X}^{*} \otimes E$.

We set

$$
\left\{\begin{array}{l}
d s_{k}^{2}=d s^{2}+\partial \bar{\partial} \chi_{k}(\Phi), \\
h_{k, m}=h \cdot a^{m} \cdot e^{-m q \chi_{k}(\Phi)},
\end{array}\right.
$$

where we put

$$
\chi_{k}(t)=\int_{\text {inf } \Phi}^{t} \lambda_{k}(u) d u
$$

letting $\lambda_{k}$ be as in Lemma 4.4 in Chapter 2 except that $c$ and $d$ are interchanged, and where we regard $\partial \bar{\partial} \chi_{k}(\Phi)$ naturally as a section of $T_{X}^{\prime} \otimes T_{X}^{\prime \prime}$.

We have

$$
\Theta_{h_{k, m}}=m \Theta_{a}+\Theta_{h}+m q \partial \bar{\partial} \chi_{k}(\Phi) .
$$

Since $\chi_{k}(t)$ is a convex increasing function, $\partial \bar{\partial} \chi_{k}(\Phi)$ is a positive semi-definite form. Hence by Lemma 1.5 , at any point $x \in X_{c}-X_{d-\delta}$, the eigenvalues $\Gamma_{1}^{k} \geqq \cdots \geqq \Gamma_{n}^{k}$ of $\Theta_{a}+q \partial \bar{\partial} \chi_{k}(\Phi)$ with respect to $d s_{k}^{2}$ satisfy

$$
\Gamma_{i}^{k}>q, \quad 1 \leqq i \leqq n-q+1 .
$$

Clearly, we have

$$
\Gamma_{i}^{k}>-1, \quad n-q+1<i \leqq n .
$$

On the other hand, letting $\omega$ and $\omega_{k}$ be the fundamental forms associated to $d s^{2}$ and $d s_{k}^{2}$, respectively, we have

$$
d \omega_{k}=d \omega,
$$

so $e\left(d \omega_{k}\right)$ is bounded. Hence similarly as in the pseudoconvex case of Theorem 4.2 in Chapter 2, we conclude that, for sufficiently large $m,\left(X_{d}, X_{c}\right)$ is a pseudoRunge pair with respect to $K_{X}^{*} \otimes E \otimes B^{m}$ at bi-degrees $(n, p), p \geqq q-1$, whence follows the theorem.

q.e.d.

The following definition is essentially due to Griffiths [8].

Definition 1.6. A hermitian vector bundle $(E, h)$ over a complex manifold $X$ of dimension $n$ is said to be weakly $q$-positive if, for any point $x \in X$ and for any $v \in E_{x}-\{0\}, h\left(\Theta_{h} v\right)(\bar{v})$ is a hermitian form on $T_{X, x}$ having at least $n-q+1$ positive eigenvalues. Here we put $h\left(\Theta_{h} v\right)(\bar{v})=e\left(h\left(e\left(\Theta_{h}\right) v\right)\right) \bar{v}$ for simplicity.

Let $\pi: P(E) \rightarrow X$ be the bundle of projective spaces associated to $E \rightarrow X$. Over $P(E)$ there is a tautological line bundle $L(E)$ whose fiber $L(E)_{\xi}(\xi \in P(E))$ 
is the line $[\xi] \rightarrow E_{\pi(\xi)}$. By Leray's theorem, we have a canonical isomorphism

$$
H^{p}\left(X, \mathscr{S} \otimes E^{(m)}\right) \cong H^{p}\left(P\left(E^{*}\right), \pi^{*} \mathscr{S} \otimes L^{m}\right), \quad m \geqq 0,
$$

where $L=\left(L\left(E^{*}\right)\right)^{*}$ and $\mathscr{S}$ is any locally free coherent analytic sheaf over $X$.

Proposition 1.7. If $(E, h)$ is weakly q-positive, then $L \rightarrow P\left(E^{*}\right)$, with the induced metric, is q-positive.

Proof. See (2.36) in [9].

From Proposition 1.7, combined with the canonical isomorphism (10), we obtain the following corollaries to Theorem 1.3.

Corollary 1.8. Let $(E, h)$ be a hermitian vector bundle over a weakly 1complete manifold $X$. Assume that $(E, h)$ is weakly q-positive outside a compact subset $K \subset X_{c}$. Then, for any holomorphic vector bundle $F$ over $X$, there is an integer $m_{0}$ such that $H^{p}\left(X_{c}, F \otimes E^{(m)}\right), m>m_{0}$, are finite dimensional and the natural restriction map

$$
H^{p}\left(X_{c}, F \otimes E^{(m)}\right) \longrightarrow H^{p}\left(X_{d}, F \otimes E^{(m)}\right), \quad c>d, m>m_{0}
$$

is bijective, if $p \geqq q$ and $X_{d} \supset K$.

Corollary 1.9. Let the situations be as above. If moreover $K=\emptyset$, then for any holomorphic vector bundle $F$ over $X$, there is an integer $m_{0}$ such that

$$
H^{p}\left(X_{c}, F \otimes E^{(m)}\right)=0, \quad \text { for } \quad p \geqq q, m>m_{0} .
$$

Remark. Corollary 1.9, which is along the line of Nakano-Hironaka (cf. [19]), is a generalization of the coarse vanishing theorems for compact complex manifolds obtained by Andreotti-Grauert [3] and Griffiths [8], whose original form is found in Kodaira [13].

As a generalization of the dual of Corollary 1.9 for compact manifolds, we obtain the following

Theorem 1.10. Let $(E, a)$ be a hermitian vector bundle of rank $s$ over a weakly 1-complete manifold $X$. Assume that $(E, a)$ is q-negative outside a compact subset $K \subset X_{d}$ and that the rank of $\partial \bar{\partial}\left(e^{\Phi}\right)$ is everywhere $\leqq r$. Then, for any' $c \in \mathbf{R}$ with $X_{c} \supset X_{d}$, and for any vector bundle $F$ over $X$, there is an integer $m_{0}$ such that the natural restriction maps

$$
H^{p}\left(X_{c}, F \otimes E^{(m)}\right) \longrightarrow H^{p}\left(X_{d}, F \otimes E^{(m)}\right),
$$

are bijective and $H^{p}\left(X_{c}, F \otimes E^{(m)}\right)$ are finite dimensional, if $X_{d} \rightarrow K, m>m_{0}$, and $p \leqq n-r-q-s+1$. 
Proof. By (2.36) in [9], $L=\left(L\left(E^{*}\right)\right)^{*}$ is $q+s-1$ negative outside $K$. Hence by the isomorphism (10), we may assume that $E$ is a line bundle.

Let $\chi_{k}$ be as before. By hypothesis, the rank of $\partial \bar{\partial} \chi_{k}(\Phi)$ is $\leqq r$. We put

$$
\left\{\begin{array}{l}
d s_{k}^{2}=d s^{2}+\partial \bar{\partial} \chi_{k}(\Phi) \\
h_{k, m}=h a^{m} e^{-m \chi_{k}(\Phi)},
\end{array}\right.
$$

where $d s^{2}$ is so chosen that at least $n-q+1$ eigenvalues of $\Theta_{a}$ are less than $-2(q+r)$ and other eigenvalues are less than 1 , both outside a fixed $X_{d-\delta}$ such that $K \subset X_{d-\delta} \subset X_{d}$, and $h$ is a hermitian metric along the fibers of $F$. Then, at any point $x \in X_{c}-X_{d-\delta}$, the eigenvalues $\Gamma_{1}^{k} \geqq \cdots \geqq \Gamma_{n}^{k}$ of $\Theta_{a}+\partial \bar{\partial} \chi_{k}(\Phi)$ with respect to $d s_{k}^{2}$ satisfy

$$
\left\{\begin{array}{l}
\Gamma_{i}^{k} \leqq 2, \quad 1 \leqq i \leqq q+r-1 \\
\Gamma_{i}^{k} \leqq-2(q+r), \quad q+r-1<i \leqq n
\end{array}\right.
$$

The rest of the proof is the same as in the proof of Theorem 1.3. q.e.d.

In particular, if $K=\varnothing$, then we have a vanishing theorem as Corollary 1.9.

Remark 1. By the same argument we can prove the corresponding coarse vanishing theorems (see Corollary 1.4) for semi-positive bundles (resp. seminegative bundles) of type $q$ (for the definition see the next chapter) over strongly $q$-pseudoconvex (resp. strongly $q$-pseudoconcave) manifolds.

Remark 2. It will be interesting to know whether

$$
\operatorname{dim} H^{p}\left(X_{c}, F \otimes E^{(m)}\right), p \geqq q,
$$

are at most of polynomial growth of degree $n$ with respect to $m$, where $E$ is as in Theorem 1.3. The corresponding result for strongly $q$-pseudoconvex manifolds and strongly $q$-pseudoconcave manifolds has already been obtained by $\mathrm{D}$. Leistner [14].

\section{§ 2. Precise Isomorphism Theorems}

Let $(E, h)$ be a hermitian vector bundle over a complex manifold $X$ of dimension $n$. $(E, h)$ is said to be semi-positive (resp. semi-negative) if, for any point $x \in X,(h \otimes i d) \Theta_{h}$ is a positive semi-definite (resp. negative semi-definite) hermitian form on $\left(E \otimes T_{X}\right)_{x} . \quad(E, h)$ is said to be semi-positive of type $q$ (resp. semi-negative of type $q)$, if $(E, h)$ is both semi-positive (resp. semi-negative) and $q$-positive (resp. $q$-negative). 
Proposition 2.1. Let $(E, h)$ be a semi-positive hermitian vector bundle of type $q$ over a complex manifold $X$ with a hermitian metric $d s^{2}$. Then, at any point $x \in X$,

$$
\sqrt{-1}\left\langle e\left(\Theta_{h}\right) \Lambda f, f\right\rangle \geqq \gamma(x)\langle f, f\rangle \quad \text { for } f \in C^{n, p}(X, E), \quad p \geqq q,
$$

where

$$
\gamma(x):=\max _{\substack{S_{x} \triangleleft T_{X}, x \\ \mathrm{dim} S_{x}=n-q+1}} \min _{\substack{f \in E_{x} \otimes S_{x} \\ f \neq 0}} \frac{\left((h \otimes \mathrm{id})\left(\Theta_{h} f\right)\right)(f)}{\langle f, f\rangle} .
$$

Proof. Let $\left(\tau_{1}^{*}, \ldots, \tau_{n}^{*}\right)$ be an orthonormal basis of $T_{X, x}$ with respect to $d s^{2}$ such that, if $S_{x}$ is the linear subspace of $T_{X, x}$ spanned by $\tau_{1}^{*}, \ldots, \tau_{n-q+1}^{*}$, we have

$$
\gamma(x)=\min _{\substack{f \in E_{x} \otimes S_{x} \\ f \neq 0}} \frac{\left((h \otimes \mathrm{id})\left(\Theta_{h} f\right)\right)(f)}{\langle f, f\rangle} .
$$

By direct computation, we have

$$
\begin{aligned}
& \sqrt{-1}\left\langle e\left(\tau_{j_{\alpha}} \wedge \bar{\tau}_{\beta}\right) \wedge \tau_{1} \wedge \cdots \wedge \tau_{n} \wedge \bar{\tau}_{j_{1}} \wedge \cdots \wedge \bar{\tau}_{j_{p}}\right. \\
& \left.\tau_{1} \wedge \cdots \wedge \tau_{n} \wedge \bar{\tau}_{j_{1}} \wedge \cdots \wedge \bar{\tau}_{j_{\alpha-1}} \wedge \bar{\tau}_{\beta} \wedge \bar{\tau}_{j_{\alpha+1}} \wedge \cdots \wedge \bar{\tau}_{j_{p}}\right\rangle \\
& \quad=1, \quad p \geqq 1,
\end{aligned}
$$

where $\left(\tau_{1}, \ldots, \tau_{n}\right)$ denotes the dual basis of $\left(\tau_{1}^{*}, \ldots, \tau_{n}^{*}\right)$. We set

$$
\sqrt{-1}\left\langle e\left(\Theta_{h}\right) \Lambda \varphi, \varphi\right\rangle=\sum_{1 \leqq \alpha, \beta \leqq n} \Theta_{\alpha \beta}\left(\varphi_{\alpha}, \varphi_{\beta}\right),
$$

where

$$
\varphi=\sum_{1 \leqq \alpha \leqq n} \varphi_{\alpha} \tau_{1} \wedge \cdots \wedge \tau_{n} \wedge \bar{\tau}_{\alpha}, \quad \varphi_{\alpha} \in E_{x},
$$

and $\Theta_{\alpha \beta}$ is the coefficient of $\tau_{\alpha} \wedge \bar{\tau}_{\beta}$ in $\sqrt{-1} \Theta_{l}$. For any multi-index $J=\left(j_{1}, \ldots, j_{p}\right)$ with $j_{1}<\cdots<j_{p}$ and for any $J^{\prime}=\left(j_{1}, \ldots, \check{j}_{\alpha}, \ldots, j_{p}\right)$, we put

$$
\begin{aligned}
& f_{J^{\prime} \beta} \tau_{1} \wedge \cdots \wedge \tau_{n} \wedge \bar{\tau}_{j_{1}} \wedge \cdots \wedge \check{\bar{\tau}}_{j_{\alpha}} \wedge \cdots \wedge \bar{\tau}_{j_{p}} \wedge \bar{\tau}_{\beta} \\
& \quad=f_{j_{1}^{\prime} \cdots j_{p}^{\prime}} \tau_{1} \wedge \cdots \wedge \tau_{n} \wedge \bar{\tau}_{j_{1}^{\prime}} \wedge \cdots \wedge \bar{\tau}_{j_{p}^{\prime}}, \quad j_{1}^{\prime}<\cdots<j_{p}^{\prime},
\end{aligned}
$$

where $\left\{j_{1}^{\prime}, \ldots, j_{p}^{\prime}\right\}=\left\{j_{1}, \ldots, \check{j}_{\alpha}, \ldots, j_{p}, \beta\right\}$ and

$$
f=\sum_{j_{1}<\cdots<j_{p}} f_{j_{1} \cdots j_{p}} \tau_{1} \wedge \cdots \wedge \tau_{n} \wedge \bar{\tau}_{j_{1}} \wedge \cdots \wedge \bar{\tau}_{j_{p}}, f_{j_{1} \cdots j_{p}} \in E_{x} .
$$

Then, by (4),

$$
\begin{aligned}
& \sqrt{-1}\left\langle e\left(\Theta_{h}\right) \Lambda f, f\right\rangle \\
& =\sum_{\left|J^{\prime}\right|=p-1} \sum_{1 \leqq \alpha, \beta \leqq n} \Theta_{\alpha \beta}\left(f_{J^{\prime} \alpha}, f_{J^{\prime} \beta}\right) \\
& =\sum_{\left|J^{\prime}\right|=p-1} \sum_{1 \leqq \alpha, \beta \leqq n-p+1} \Theta_{i_{\alpha} i_{\beta}}\left(f_{J^{\prime} i_{\alpha}}, f_{J^{\prime} i_{\beta}}\right)
\end{aligned}
$$




$$
\begin{aligned}
& \geqq \sum_{\left|J^{\prime}\right|=p-1} \sum_{1 \leqq \alpha, \beta \leqq n-p+1} \Theta_{\alpha \beta}\left(f_{J^{\prime} \alpha}, f_{J^{\prime} \beta}\right) \\
& \geqq \gamma(x) \sum_{\left|J^{\prime}\right|=p-1}\left\langle\sum_{\alpha=1}^{n-p+1} f_{J^{\prime} \alpha} \otimes \tau_{\alpha}^{*}, \sum_{\alpha=1}^{n-p+1} f_{J^{\prime} \alpha} \otimes \tau_{\alpha}^{*}\right\rangle .
\end{aligned}
$$

Hence,

$$
\begin{aligned}
& \sqrt{-} 1\left\langle e\left(\Theta_{h}\right) \Lambda f, f\right\rangle \\
& \geqq \gamma(x) \sum_{\alpha=1}^{n-p+1}\left(\sum_{J \ni \alpha}\left\langle f_{J}, f_{J}\right\rangle\right) \\
& \geqq \gamma(x)\langle f, f\rangle .
\end{aligned}
$$

The following theorem has been proved by Ohsawa [23] and by NakanoRhai [22] for $q=1$ ( $r a n k E=1$ is assumed in [23]). The original form where $K=\varnothing$ and $q=1$ was proved by Nakano [17], [19], [20], [21] and Kazama [12], and reproved by Suzuki [27].

Theorem 2.2. Let $X$ be a weakly 1-complete manifold, and let $(E, h)$ be a hermitian vector bundle over $X$. Assume that $(E, h)$ is semi-positive of type $q$ outside a compact subset $K \subset X_{d}$ and $d s^{2}$ is Kählerian outside $K$. Then $H^{p}\left(X, \Omega^{n}(E)\right)$ are finite dimensional and the natural restriction maps

$$
H^{p}\left(X, \Omega^{n}(E)\right) \longrightarrow H^{p}\left(X_{d}, \Omega^{n}(E)\right)
$$

are bijective, if $p \geqq q$. In particular, if $K=\emptyset$,

$$
H^{p}\left(X, \Omega^{n}(E)\right)=0, \quad \text { for } \quad p \geqq q .
$$

Proof. For any $c>d,\left(X_{d}, X_{c}\right)$ is a pseudo-Runge pair with respect to $E$ at bi-degrees $(n, p), p \geqq q-1$. In fact we have only to put

$$
\left\{\begin{array}{l}
d s_{k}^{2}=d s^{2}+\partial \bar{\partial} \chi_{k}(\Phi) \\
h_{k}=h e^{-\chi_{k}(\Phi)}
\end{array}\right.
$$

Then, in virtue of Proposition 2.1, we can prove the basic estimates. The rest of the proof is the same as in the proof of Theorem 1.3.

Proposition 2.3. Let $(E, h)$ be a semi-negative vector bundle of type $q$ over a complex manifold $X$ of dimension $n$ with a hermitian metric $d s^{2}$. Then, at any point $x \in X$,

$$
\begin{aligned}
& -\sqrt{-1}\left\langle\Lambda e\left(\Theta_{h}\right) f, f\right\rangle \geqq \delta(x)\langle f, f\rangle, \\
& f \in C^{0, p}(X, E), \quad p \leqq n-q .
\end{aligned}
$$

Here, 


$$
\delta(x):=\min _{\substack{S_{x^{4}} T_{X, x}, x+1 \\ \operatorname{dim} S_{x}=n-q+1}} \max _{\substack{f \in E_{x} \otimes S_{x} \\ f \pm 0}} \frac{\left((h \otimes \mathrm{id})\left(\Theta_{h} f\right)\right)(f)}{\langle f, f\rangle} .
$$

Proof. Similar to Proposition 2.1.

As a dual of Theorem 2.2, we obtain

Theorem 2.4. Let $X$ be a weakly' 1-complete manifold and let $(E, h)$ be a hermitian vector bundle over $X$. Assume that $(E, h)$ is semi-negative of type $q$ outside a compact subset $K \subset X_{d}, X$ is Kählerian outside $K$, and that $\partial \bar{\partial}\left(e^{\Phi}\right)$ is of rank $\leqq r$. Then $H^{p}(X, E)$ are finite dimensional and the natural restriction maps

$$
H^{p}(X, E) \longrightarrow H^{p}\left(X_{d}, E\right)
$$

are bijective, if $p \leqq n-q-r$. In particular, if $K=\emptyset$, then

$$
H^{p}(X, E)=0, \quad \text { for } \quad p \leqq n-q-r .
$$

Proof. Similar to Theorem 2.2.

The original form of the following theorem has been proved by AkizukiNakano [2] and Girbau [7]. The present form has been partially proved by Nakano [20], Abdelkader [1], Ohsawa [24], and Takegoshi-Ohsawa [29].

Theorem 2.5. Let $(B, a)$ be a hermitian line bundle over a weakly 1complete manifold $X$. Assume that $(B, a)$ is semi-positive of type q outside a compact subset $K \subset X_{d}$ and that $X$ is Kählerian outside $K$. Then $H^{t}\left(X, \Omega^{s}(B)\right)$ are finite dimensional and the natural restriction maps

$$
H^{t}\left(X, \Omega^{s}(B)\right) \longrightarrow H^{t}\left(X_{d}, \Omega^{s}(B)\right),
$$

are bijective, if $s+t \geqq n+q$. In particular, if $K=\varnothing$, then

$$
H^{t}\left(X, \Omega^{s}(B)\right)=0, \quad \text { for } s+t \geqq n+q .
$$

Proof. Let $c>d$ and

$$
\left\{\begin{array}{l}
d s_{k}^{2}=\varepsilon d s^{2}+\partial \bar{\partial} \chi_{k}(\Phi)+\Theta_{a} \\
a_{k}=a e^{-\chi_{k}(\Phi)}
\end{array}\right.
$$

where $\varepsilon$ is a positive number which is determined later. We have to prove that $\left(X_{d}, X_{c}\right)$ is a pseudo-Runge pair with respect to the above metrics.

It is clear that $(*)$ is satisfied.

Let $x \in X_{c}-X_{d-\delta}$ be any point $\left(X_{d} \supset X_{d-\delta} \supset K\right)$. We set 


$$
\left\{\begin{array}{l}
d s^{2}=\sum_{i=1}^{n} \tau_{i}^{k} \otimes \bar{\tau}_{i}^{k} \\
d s_{k}^{2}=\sum_{i=1}^{n}\left(\varepsilon+\Gamma_{i}^{k}\right) \tau_{i}^{k} \otimes \bar{\tau}_{i}^{k} \quad \text { at } \quad x .
\end{array}\right.
$$

Let $\Gamma_{1} \geqq \cdots \geqq \Gamma_{n} \geqq 0$ be the eigenvalues of $\Theta_{a}$ at $x$ with respect to $d s^{2}$. Then we have

$$
\Gamma_{i}^{k} \geqq \Gamma_{i}
$$

Let

$$
\begin{aligned}
& f=\sum_{\substack{i_{1}<\cdots<i_{s} \\
j_{1}<\cdots<j_{t} \\
i_{1} \cdots i_{s} \bar{J}_{1} \cdots \bar{j}_{t}}} \tau_{i_{1}}^{k} \wedge \cdots \wedge \tau_{i_{s}}^{k} \wedge \bar{\tau}_{j_{1}}^{k} \wedge \cdots \wedge \bar{\tau}_{j_{t}}^{k}, \\
& f_{i_{1} \cdots i_{s} \bar{j}_{1} \cdots \bar{j}_{t}}^{k} \in B_{x} .
\end{aligned}
$$

Then, (cf. [7])

$$
\begin{aligned}
& \sqrt{-1}\left(e\left(\Theta_{a_{k}}\right) \Lambda_{k}-\Lambda_{k} e\left(\Theta_{a_{k}}\right)\right) f \\
& =\sum_{\substack{i_{1}<\cdots<i_{s} \\
j_{1}<\cdots<j_{t}}}\left(\sum_{\alpha=1}^{s} \frac{\Gamma_{i_{\alpha}}^{k}}{\Gamma_{i_{\alpha}}^{k}+\varepsilon}+\sum_{\beta=1}^{t} \frac{\Gamma_{j \beta}^{k}}{\Gamma_{j_{\beta}}^{k}+\varepsilon}-\sum_{\gamma=1}^{n} \frac{\Gamma_{\gamma}^{k}}{\Gamma_{\gamma}^{k}+\varepsilon}\right) \\
& \quad \cdot \prod_{\alpha=1}^{s} \frac{1}{\Gamma_{i_{\alpha}}^{k}+\varepsilon} \prod_{\beta=1}^{t} \frac{1}{\Gamma_{j_{\beta}}^{k}+\varepsilon} \\
& \quad \cdot f_{i_{1} \cdots i_{s} \bar{j}_{1} \cdots j_{t}} \tau_{i_{1}} \wedge \cdots \wedge \tau_{i_{s}} \wedge \bar{\tau}_{j_{1}} \wedge \cdots \wedge \bar{\tau}_{j_{t}} .
\end{aligned}
$$

If $s+t \geqq n+q$, then

$$
\begin{aligned}
& \sum_{\alpha=1}^{s} \frac{\Gamma_{i_{\alpha}}^{k}}{\Gamma_{i_{\alpha}}^{k}+\varepsilon}+\sum_{\beta=1}^{t} \frac{\Gamma_{j_{\beta}}^{k}}{\Gamma_{j_{\beta}}^{k}+\varepsilon}-\sum_{\gamma=1}^{n} \frac{\Gamma_{\gamma}^{k}}{\Gamma_{\gamma}^{k}+\varepsilon} \\
& \geqq \frac{(n-q+2) \Gamma_{n-q+1}^{k}}{\Gamma_{n-q+1}^{k}+\varepsilon}-\sum_{\gamma=1}^{n-q+1} \frac{\Gamma_{\gamma}^{k}}{\Gamma_{\gamma}^{k}+\varepsilon} \quad \text { (cf. [29]). }
\end{aligned}
$$

Hence, if $2 n \varepsilon<\inf _{x \in X_{c}-X_{d-\delta}} \Gamma_{n-q+1}$,

$$
\begin{aligned}
& \sqrt{-1}\left\langle\left(e\left(\Theta_{a_{k}}\right) \Lambda_{k}-\Lambda_{k} e\left(\Theta_{a_{k}}\right)\right) f, f\right\rangle \\
& \geqq \frac{n+q-1}{2 n+1}\langle f, f\rangle, \quad \text { if } \quad s+t \geqq n+q,
\end{aligned}
$$

where the inner products are with respect to $d s_{k}^{2}$ and $a_{k}$. Therefore, similarly as before, we obtain the corresponding basic estimates. The verification of $(* * *)$ is the same as in the proof of Theorem 1.3.

q.e.d.

Similarly we obtain

Theorem 2.6. Let $(B, a)$ be a hermitian line bundle over a weakly 1complete manifold $X$. Assume that $(B, a)$ is semi-negative of type q outside a 
compact subset $K \subset X_{d}, X$ is Kählerian outside $K$, and the rank of $\partial \bar{\partial}\left(e^{\Phi}\right)$ is $\leqq r$. Then $H^{t}\left(X, \Omega^{s}(B)\right)$ are finite dimensional and the natural restriction maps

$$
H^{t}\left(X, \Omega^{s}(B)\right) \longrightarrow H^{t}\left(X_{d}, \Omega^{s}(B)\right)
$$

are bijective, if $s+t \leqq n-q-r$. In particular, if $K=\emptyset$,

$$
H^{t}\left(X, \Omega^{s}(B)\right)=0, \quad \text { for } \quad s+t \leqq n-q-r .
$$

Proof. Let $c>d$ and

$$
\left\{\begin{array}{l}
d s_{k}^{2}=\varepsilon d s^{2}+\partial \bar{\partial} \chi_{k}(\Phi)-\Theta_{a} \\
a_{k}=a e^{-i \chi_{k}(\Phi)},
\end{array}\right.
$$

where $\varepsilon$ is a positive number which is determined later. We shall show that $\left(X_{d}, X_{c}\right)$ is a pseudo-Runge pair with respect to the above metrics. It is clear that $(*)$ is satisfied. Let $x \in X_{c}-X_{d-\delta}$ be any point, where a positive number $\delta$ is so chosen that $X_{d} \supset X_{d-\delta} \supset K$. We have

$$
\begin{aligned}
\Theta_{a_{k}} & =\Theta_{a}+\partial \bar{\partial} \chi_{k}(\Phi) \\
& =-d s_{k}^{2}+\varepsilon d s^{2}+(1+\varepsilon) \partial \bar{\partial} \chi_{k}(\Phi) .
\end{aligned}
$$

Let $f \in\left(B \otimes T_{X}^{s, t}\right)_{x}$, then

$$
\begin{aligned}
\left\langle\sqrt{-1}\left(e\left(\Theta_{a_{k}}\right) \Lambda_{k}-\Lambda_{k} e\left(\Theta_{a_{k}}\right)\right) f, f\right\rangle_{k} \\
=\left\langle\sqrt{-1}\left(-e\left(d s_{k}^{2}\right) \Lambda_{k}+\Lambda_{k} e\left(d s_{k}^{2}\right)\right) f, f\right\rangle_{k} \\
\quad+\left\langle\sqrt{-1}\left(e\left(\varepsilon d s^{2}+(1+\varepsilon) \partial \bar{\partial} \chi_{k}(\Phi)\right) \Lambda_{k}-\Lambda_{k} e\left(\varepsilon d s^{2}+(1+\varepsilon) \partial \bar{\partial} \chi_{k}(\Phi)\right) f, f\right\rangle_{k}\right. \\
=(n-s-t)\langle f, f\rangle_{k}+\left\langle\sqrt { - 1 } \left( e\left(\varepsilon d s^{2}+(1+\varepsilon) \partial \bar{\partial} \chi_{k}(\Phi)\right) \Lambda_{k}\right.\right. \\
\left.\left.\quad-\Lambda_{k} e\left(\varepsilon d s^{2}+(1+\varepsilon) \partial \bar{\partial} \chi_{k}(\Phi)\right)\right) f, f\right\rangle_{k}, \quad \text { at } \quad x .
\end{aligned}
$$

Here we denote by $\langle,\rangle_{k}$ the pointwise inner product with respect to $d s_{k}^{2}$ and $a_{k}$. We put

$$
\left\{\begin{array}{l}
d s_{k}^{2}=\sum_{i=1}^{n} \tau_{i}^{k} \otimes \bar{\tau}_{i}^{k} \\
\varepsilon d s^{2}+(1+\varepsilon) \partial \bar{\partial} \chi_{k}(\Phi)=\sum_{i=1}^{n}\left(1+\gamma_{i}^{k}(\varepsilon)\right) \tau_{i}^{k} \otimes \bar{\tau}_{i}^{k}, \quad \text { at } \quad x .
\end{array}\right.
$$

Here $\gamma_{i}^{k}(\varepsilon)$ are the eigenvalues of $\Theta_{a}+\varepsilon \partial \partial \bar{\partial} \chi_{k}(\Phi)$ with respect to $d s_{k}^{2}$, and we arrange them as follows:

$$
\gamma_{1}^{k}(\varepsilon) \leqq \cdots \leqq \gamma_{n}^{k}(\varepsilon) .
$$

It is easily seen that $\gamma_{i}^{k}(\varepsilon) \leqq \varepsilon$ and that

$$
-1 \leqq \gamma_{i}^{k}(\varepsilon) \leqq-1+C \varepsilon \text { for } 1 \leqq i \leqq n-q+1-r,
$$


where $C$ is a constant which does not depend on $k$ or $x$. Hence,

$$
\begin{aligned}
& \sum_{\alpha=1}^{s}\left(1+\gamma_{i_{\alpha}}^{k}(\varepsilon)\right)+\sum_{\beta=1}^{t}\left(1+\gamma_{j_{\beta}}^{k}(\varepsilon)\right)-\sum_{u=1}^{n}\left(1+\gamma_{u}^{k}(\varepsilon)\right) \\
& \left(=s+t-n+\sum_{\alpha=1}^{s} \gamma_{i_{\alpha}}^{k}(\varepsilon)+\sum_{\beta=1}^{t} \gamma_{j_{\beta}}^{k}(\varepsilon)-\sum_{u=1}^{n} \gamma_{u}^{k}(\varepsilon)\right) \\
& \geqq s+t-n-s-t+n-q+1-r-\{C(n-q+1-r)+q+r-1\} \varepsilon \\
& =-q-r+1-\{C(n-q+1-r)+q+r-1\} \varepsilon .
\end{aligned}
$$

Combining (23) with (27), we obtain the basic estimate for $s+t \leqq n-q-r$. The rest of the proof is the same as in the proof of Theorem 1.3.

q.e.d.

A hermitian vector bundle $(E, h)$ over a complex manifold $X$ of dimension $n$ is said to be weakly semi-positive if, for any point $x \in X$ and for any $v \in E_{x}-\{0\}$, $h\left(\Theta_{h}(v)\right)(\bar{v})$ is a positive semi-definite hermitian form on $T_{X, x}$. If the rank of $h\left(\Theta_{h}(v)\right)(\bar{v})$ is at least $n-q+1$, then $(E, h)$ is said to be weakly semi-positive of type $q$. This definition is originally due to Griffiths [8] (cf. also Skoda [26]).

Let $P\left(E^{*}\right)$ and $L$ be as in Section 1 . Then we have the following isomorphism (cf. Le Potier, C. R. Acad. Sc. Paris, 276 (1973) pp. 535-537).

$$
H^{t}\left(X, \Omega^{s}(E)\right) \longrightarrow H^{t}\left(P\left(E^{*}\right), \Omega^{s}(L)\right) \text {. }
$$

From (2.36) in [9], $L$ is semi-positive of type $q$ if $E$ is weakly semi-positive of type $q$. Thus combining the isomorphism (21) with Theorem 2.5, we obtain

Theorem 2.7. Let $(E, h)$ be a hermitian vector bundle of rank $r$ over a weakly 1-complete manifold $X$. Assume that $(E, h)$ is weakly semi-positive of type $q$ outside a compact subset $K \subset X_{d}$ and there is a Kähler metric on $X-K$. Then $H^{t}\left(X, \Omega^{s}(E)\right)$ are finite dimensional and the natural restriction maps

$$
H^{t}\left(X, \Omega^{s}(E)\right) \longrightarrow H^{t}\left(X_{d}, \Omega^{s}(E)\right)
$$

are bijective, if $s+t \geqq n+r+q-1$. In particular, if $K=\emptyset$,

$$
H^{t}\left(X, \Omega^{s}(E)\right)=0, \quad \text { for } \quad s+t \geqq n+r+q-1 .
$$

The counterpart of Theorem 2.6 is left to the reader.

The relation between semi-positivity and weak semi-positivity, except for the trivial implication, has been first revealed by Demailly and Skoda [5]. We restate here their theorem as follows.

Theorem 2.8. If $(E, h)$ is weakly semi-positive (resp. weakly 1-positive), then $((\operatorname{det} E) \otimes E,(\operatorname{det} h) \otimes h)$ is semi-positive (resp. 1-positive). 
It follows trivially from their inequality

$$
\sum_{j, k, l, m} a_{j k l n} x_{j l} \bar{x}_{k m}+\sum_{j, k, l, m} a_{j j l m} x_{k l} \bar{x}_{k m} \geqq \sum_{j, l, m} a_{j j l m} x_{j l} \bar{x}_{j m}
$$

(cf.p. 307 in [5]) that if $(E, h)$ is weakly semi-positive of type $q$, then $((\operatorname{det} E) \otimes E$, $(\operatorname{det} h) \otimes h)$ is semi-positive of type $q$.

Remark 1. In the above theorems we have proved also approximation theorems and harmonic representation theorems. From the harmonic representation theorem, we can deduce cohomology vanishing theorems under somewhat weaker assumptions on the curvature form of $(E, h)$ (cf. Takegoshi [28]).

Remark 2. Let $\pi: X \rightarrow \mathbf{C}^{N}$ be a proper holomorphic map from a weakly 1-complete manifold $X$ of dimension $n$. Assume that $X$ is embeddable into $\mathbb{P}^{N^{\prime}}$ as a locally closed analytic submanifold, and that $\operatorname{dim} \pi^{-1}(x) \leqq n-r$ for $x \neq 0$. Then the latter part of Theorem 2.5 (Nakano's vanishing theorem), combined with Hodge-Lieberman-Rossi-Fujiki's decomposition theorem for strongly pseudoconvex manifolds, (cf. [6], [15]) implies that the decomposition of $H^{p}(X, \mathbb{C})$ into the direct sum of $H^{t}\left(X, \Omega^{s}\right)$ and the symmetry $\operatorname{dim} H^{t}\left(X, \Omega^{s}\right)$ $=\operatorname{dim} H^{s}\left(X, \Omega^{t}\right)$ are valid for degrees $s+t, p \geqq 2 n-r+1$. Therefore the topology of the degenerate set of a holomorphic map from a projective variety is very restricted. Such phenomenon can be observed on Kähler manifolds, too (cf. [25]), which may suggest a further meaning of our isomorphism theorems.

\section{Appendix}

Let the notations be as in Chapter 1. We shall prove here Theorem 1.3 following [31] and [32]. We have to deal with operators on $T_{X}^{r}$ rather than those on $T_{X}^{p, q}$, so we regard $L, \Lambda$, and so on, as operators on $T_{X}^{r}$.

Definition 1. An $r$-form $u$ is said to be primitive if $\Lambda u=0$.

From now on $r$-forms (resp. primitive $r$-forms) are denoted by $f^{r}$ (resp. $\left.u^{(r)}\right)$, unless otherwise stated. For the proofs of Theorem 2 to Theorem 6, the reader is referred to [31] and [32].

Theorem 2. i) If $u^{(r)}$ is a nonzero primitive form, then $r \leqq n$.

ii) If $u^{(r)}$ is primitive with $r \leqq n$, then

$$
\left\{\begin{array}{l}
L^{n-r+1} u^{(r)}=0 \\
\Lambda^{k} L^{k} u^{(r)}=\frac{k !(n-r) !}{(n-r-k) !} u^{(r)} \quad \text { for } \quad k \leqq n-r .
\end{array}\right.
$$


Theorem 3. i) For any fr with $r \leqq n$,

$$
f^{r}=u^{(r)}+L u^{(r-2)}+\cdots+L^{k} u^{(r-2 k)}, \quad k \leqq[r / 2] .
$$

ii) For any $f^{r}$ with $r>n$,

$$
\begin{aligned}
& f^{r}=L^{r-n} u^{(2 n-r)}+L^{r-n+1} u^{(2 n-r-2)}+\cdots+L^{r-n+k} u^{(2 n-r-2 k)}, \\
& k \leqq[n-(r / 2)] .
\end{aligned}
$$

Furthermore the decompositions $i$ ) and ii) are unique.

Corollary 4. If $L^{n-r+k} f^{r}=0, n-r+k \geqq 0$, then $u^{(i)}=0, i \leqq r-2 k$, where $u^{(i)}$ are as in i) or ii).

Corollary 5. There is an operator $P(n, r, i)$ belonging to the algebra generated by $\Lambda$ and $L$ over the field of rational numbers, satisfying

$$
P(n, r, i) f^{r}=u^{(i)},
$$

where $u^{(i)}$ are as in i) or ii).

Theorem 6. For any $u^{(r)}$,

$$
\bar{*} L^{k} u^{(r)}= \begin{cases}(-1)^{\frac{r(r+1)}{2}} \frac{k !}{(n-r-k !)} L^{n-r-k} \bar{C} u^{(r)}, \quad k \leqq n-r \\ 0, \quad k>n-r .\end{cases}
$$

From these theorems we can deduce

Proposition 7. For any $u^{(p)}$ of class $C^{\infty}$,

(6)

$$
\begin{aligned}
(d \Lambda & -\Lambda d) L^{k} u^{(p)} \\
= & \left\{-C^{-1} \bar{*} d \bar{*} C+a_{n, p, k} L^{k+1} \Lambda^{3} e(d \omega) \Lambda^{k}\right. \\
& +b_{n, p, k}(\Lambda L+(k-1)(k-1-n+p)) e(d \omega) L^{k-2} \Lambda^{k} \\
& \left.+c_{n, p, k} \bar{C}^{-1} \bar{*} e(d \omega) L^{n-k-p-1} \Lambda^{k}\right\} L^{k} u^{(p)},
\end{aligned}
$$

where

(A)

$$
\begin{aligned}
a_{n, p, k}=\{2 n- & \left.2 p-k+4+\frac{(n-k-p+2)(n-k-p+1)}{k+1}\right\} \\
& \times \frac{(n-p+1)(n-p-k) !}{3 ! k !(n-p) !}
\end{aligned}
$$

(B)

$$
\begin{aligned}
& b_{n, p, k}=-\frac{(n-p-k) !}{(k-1) !(n-p) !} \\
& c_{n, p, k}=-(-1)^{\frac{p(p-1)}{2}} \frac{n-p-k}{(n-p) !},
\end{aligned}
$$

and we put $m !=0$ and $L^{m}=0$ for negative $m$. 


$$
\begin{aligned}
& (e(d \Phi) \Lambda-\Lambda e(d \Phi)) L^{k} u^{(p)} \\
& \quad=-C^{-1} \bar{*} e(d \bar{\Phi}) \bar{*} C L^{k} u^{(p)},
\end{aligned}
$$

where $\Phi$ is a (complex valued) $C^{\infty}$ function.

Admitting Proposition 7, Theorem 1.3 is proved as follows:

Proof of Theorem 1.3. Combining (6) with Theorem 3 and Corollary 5, for any $f^{r}$ of class $C^{\infty}$,

$$
(d \Lambda-\Lambda d) f^{r}=-C^{-1} \bar{*} d \bar{*} C f^{r}+T f^{r},
$$

where $T$ is a linear operator belonging to the algebra over the field of rational numbers generated by $L, \Lambda, e(d \omega), \bar{C}^{-1}$, and $\bar{*}$. Similarly we have

$$
(e(d \Phi) \Lambda-\Lambda e(d \Phi)) f^{r}=-C^{-1} \bar{*} e(d \bar{\Phi}) \bar{*} C f^{r} .
$$

Decomposing (8) and (9) into types, we have

$$
\begin{aligned}
& \begin{aligned}
& \partial \Lambda-\Lambda \partial=-\sqrt{-1}\left(\bar{*} \bar{\partial}+T_{1}\right), \\
& \begin{aligned}
\bar{\partial} \Lambda-\Lambda \bar{\partial} & =\sqrt{-1}\left(\bar{*} \partial \bar{*}+T_{2}\right) \\
& =\sqrt{-1}\left(\bar{\vartheta}+T_{2}\right),
\end{aligned} \\
& e(\partial \Phi) \Lambda-\Lambda e(\partial \Phi)=-\sqrt{-1} \bar{*} e(\bar{\partial} \bar{\Phi}) \bar{*},
\end{aligned}
\end{aligned}
$$

and

$$
e(\bar{\partial} \Phi) \Lambda-\Lambda e(\bar{\partial} \Phi)=\sqrt{-1} \bar{*} e(\partial \bar{\Phi}) \bar{*},
$$

where $T_{1}$ and $T_{2}$ are the components of $T$.

Thus in particular we have proved the latter part of (13) in Theorem 1.3.

By (12), we have

$$
\begin{gathered}
e\left(h^{-1}(\partial h)\right) \Lambda-\Lambda e\left(h^{-1}(\partial h)\right) \\
=-\sqrt{-1} \star e\left(\bar{h}^{-1}(\bar{\partial} \bar{h})\right) \bar{*} .
\end{gathered}
$$

On the other hand,

$$
\begin{aligned}
\vartheta_{E} & =-\bar{*} \bar{h}^{-1} \bar{\partial} \bar{h} \bar{*} \\
& =-\bar{*} \bar{\partial} \bar{*}-\bar{*} \bar{h}^{-1} e(\bar{\partial} \bar{h}) \bar{*} .
\end{aligned}
$$

Combining these equalities we obtain Theorem 1.3. q.e.d.

Proof of Proposition 7. We prove only (6). The proof of (7) is similar. First we note that

$$
L^{n-p+2} d u^{(p)}=d L^{n-p+2} u^{(p)}-(n-p+2) e(d \omega) L^{n-p+1} u^{(p)} .
$$


From Theorem 2 ii), the right hand side of (16) is zero, so that by Corollary 4, we have the primitive decomposition

$$
d u^{(p)}=\eta^{(p+1)}+L \eta^{(p-1)}+L^{2} \eta^{(p-3)} .
$$

Hence we have

$$
\begin{aligned}
(d \Lambda-\Lambda d) L^{k} u^{(p)} \\
= \\
=d \Lambda L^{k} u^{(p)}-\Lambda L^{k} d u^{(p)}-k \Lambda e(d \omega) L^{k-1} u^{(p)} \\
=d \Lambda L^{k} u^{(p)}-\Lambda L^{k}\left(\eta^{(p+1)}+L \eta^{(p-1)}+L^{2} \eta^{(p-3)}\right) \\
\quad-k \Lambda e(d \omega) L^{k-1} u^{(p)} .
\end{aligned}
$$

By Theorem 2 ii),

$$
\begin{aligned}
& \Lambda L^{k} u^{(p)}=-k(k-1-n+p) L^{k-1} u^{(p)}, \\
& \Lambda L^{k} \eta^{(p+1)}=-k(k-n+p) L^{k-1} \eta^{(p+1)}, \\
& \Lambda L^{k+1} \eta^{(p-1)}=-(k+1)(k-1-n+p) L^{k} \eta^{(p-1)},
\end{aligned}
$$

and

$$
L^{k+2} \eta^{(p-3)}=-(k+2)(k-2-n+p) L^{k+1} \eta^{(p-3)} .
$$

Hence,

$$
\begin{aligned}
d \Lambda L^{k} u^{(p)} \\
=-k(k-1-n+p) d L^{k-1} u^{(p)} \\
=-k(k-1-n+p)\left\{L^{k-1}\left(\eta^{(p+1)}+L \eta^{(p-1)}+L^{2} \eta^{(p-3)}\right)\right. \\
\\
\left.\quad+(k-1) e(d \omega) L^{k-2} u^{(p)}\right\} .
\end{aligned}
$$

Combining (18) with (23), we obtain

$$
\begin{aligned}
(d \Lambda & -\Lambda d) L^{k} u^{(p)} \\
= & k L^{k-1} \eta^{(p+1)}-(n-p-k+1) L^{k} \eta^{(p-1)} \\
& -(2 n-2 p-k+4) L^{k+1} \eta^{(p-3)} \\
& -\left(k \Lambda e(d \omega) L^{k-1}+k(k-1)(k-1-n+p) e(d \omega) L^{k-2} u^{(p)}\right. \\
= & k L^{k-1} \eta^{(p+1)}-(n-p-k+1) L^{k} \eta^{(p-1)} \\
& -(2 n-2 p-k+4) L^{k+1} \eta^{(p-3)} \\
& -\frac{(n-p-k) !}{(k-1) !(n-p) !}\{\Lambda L+(k-1)(k-1-n+p)\} e(d \omega) L^{k-2} \Lambda^{k} L^{k} u^{(p)}
\end{aligned}
$$

On the other hand, by Theorem 6 , we have 
(25)

$$
\begin{aligned}
- & C^{-1} \bar{*} d^{*} C L^{k} u^{(p)} \\
= & -\bar{C}^{-1} \bar{*} d \bar{*} L^{k} \bar{C} u^{(p)} \\
= & -\bar{C}^{-1} \bar{*} d \bar{*}(-1)^{\frac{p(p+1)}{2}} \frac{k !}{(n-k-p) !}{ }^{*} L^{n-k-p} u^{(p)} \\
= & -\bar{C}^{-1} \bar{*} d(-1)^{\frac{p(p-1)}{2}} \frac{k !}{(n-k-p) !} L^{n-k-p} u^{(p)} \\
= & -(-1)^{\frac{p(p-1)}{2}} \frac{k !}{(n-k-p) !} \bar{C}^{-1} \bar{*} L^{n-k-p}\left(\eta^{(p+1)}+L \eta^{(p-1)}\right. \\
& \left.+L^{2} \eta^{(p-3)}\right)+(n-k-p) e(d \omega) L^{n-k-p-1} u^{(p)} .
\end{aligned}
$$

Applying Theorem 6 again, we have

$$
\begin{array}{rl}
-C^{-1} & * d * C L^{k} u^{(p)} \\
= & -(-1)^{\frac{p(p-1)}{2}} \frac{k !}{(n-k-p) !} \bar{C}^{-1}\left\{(-1)^{\frac{(p+1)(p+2)}{2}}\right. \\
& \quad \frac{(n-k-p) !}{(k-1) !} L^{k-1} \bar{C} \eta^{(p+1)} \\
& +(-1)^{\frac{p(p-1)}{2}} \frac{(n-k-p+2) !}{(k+1) !} L^{k} \bar{C} \eta^{(p-1)} \\
& +(-1)^{\frac{(p-2)(p-3)}{2}} \frac{(n-k-p+2) !}{(k+1) !} L^{k+1} \bar{C} \eta^{(p-3)} \\
& \left.+(n-k-p) \bar{*} e(d \omega) L^{n-k-p-1} u^{(p)}\right\} \\
= & k L^{k-1} \eta^{(p+1)}-(n-p-k+1) L^{k} \eta^{(p-1)} \\
& +\frac{(n-k-p+2)(n-k-p+1)}{k+1} L^{k+1} \eta^{(p-3)} \\
& -(-1)^{\frac{p(p-1)}{2}} \frac{k !}{(n-k-p-1) !} \bar{C}^{-1} \bar{*} e(d \omega) L^{n-k-p-1} u^{(p)} \\
= & k L^{k-1} \eta^{(p+1)}-(n-p-k+1) L^{k} \eta^{(p-1)} \\
& +\frac{(n-k-p+2)(n-k-p+1)}{k+1} L^{k+1} \eta^{(p-3)} \\
& -(-1)^{\frac{p(p-1)}{2}} \bar{C}-1 \bar{*} e(d \omega) \frac{n-p-k}{(n-p) !} L^{n-k-p-1} \Lambda^{k} L^{k} u^{(p)} .
\end{array}
$$

Therefore,

$$
\begin{aligned}
(d \Lambda & -\Lambda d) L^{k} u^{(p)} \\
= & -C^{-1} \bar{*} d \bar{*} C L^{k} u^{(p)}+a_{n, p, k}^{\prime} L^{k+1} \eta^{(p-3)} \\
& +b_{n, p, k}\{\Lambda L+(k-1)(k-1-n+p)\} e(d \omega) L^{k-2} \Lambda^{k} L^{k} u^{(p)} \\
& +c_{n, p, k} \bar{C}^{-1} \bar{*} e(d \omega) L^{n-k-p-1} \Lambda^{k} L^{k} u^{(p)},
\end{aligned}
$$

where 
$\left(\mathrm{A}^{\prime}\right)$

$$
a_{n, p, k}^{\prime}=-2 n+2 p+k-4-\frac{(n-k-p+2)(n-k-p+1)}{k+1},
$$

(B)

$$
b_{n, p, k}=-\frac{(n-p-k) !}{(k-1) !(n-p) !}
$$

and

$$
c_{n, p, k}=-(-1)^{\frac{p(p-1)}{2}} \frac{n-p-k}{(n-p) !} .
$$

It remains to express $\eta^{(p-3)}$ in terms of $L^{k} u^{(p)}$. In virtue of Theorem 3 and Corollary 4 , we can put

$$
e(d \omega) u^{(p)}=v^{(p+3)}+L v^{(p+1)}+L^{2} v^{(p-1)}+L^{3} v^{(p-3)} .
$$

Since

$$
\begin{aligned}
L^{n-p+1} d u^{(p)} & =\left(L^{n-p+1} d-d L^{n-p+1}\right) u^{(p)} \\
& =-(n-p+1) L^{n-p} e(d \omega) u^{(p)},
\end{aligned}
$$

we have

$$
\begin{aligned}
L^{n-p+1} & \eta^{(p+1)}+L^{n-p+2} \eta^{(p-1)}+L^{n-p+3} \eta^{(p-3)} \\
= & -(n-p+1)\left(L^{n-p} v^{(p+3)}+L^{n-p+1} v^{(p+1)}+L^{n-p+2} v^{(p-1)}\right. \\
& \left.+L^{n-p+3} v^{(p-3)}\right) .
\end{aligned}
$$

By the uniqueness of the primitive decomposition,

$$
\eta^{(p-3)}=-(n-p+1) v^{(p-3)} .
$$

On the other hand,

$$
\begin{aligned}
v^{(p-3)} & =\frac{(n-p) !}{3 !(n-p+3) !} \Lambda^{3} L^{3} v^{(p-3)} \\
& =\frac{(n-p) !}{3 !(n-p+3) !} \Lambda^{3} e(d \omega) u^{(p)} \\
& =\frac{(n-p-k) !}{3 !(n-p+3) !} \Lambda^{3} e(d \omega) \Lambda^{k} L^{k} u^{(p)}
\end{aligned}
$$

Thus,

$$
\eta^{(p-3)}=-\frac{(n-p+1)(n-p-k) !}{3 !(n-p-3) ! k !} \Lambda^{3} e(d \omega) \Lambda^{k} L^{k} u^{(p)}
$$

Putting (33) into (27), we obtain the proposition.

q.e.d. 


\section{References}

[1] Abdelkader, O., Annulation de la cohomologie d'une variété kählérienne faiblement 1-complète à valeur dans un fibré vectoriel holomorphe semi-positif, C.R.Acad.Sc. Paris, 290 (1980), 75-78.

[2] Akizuki, Y. and Nakano, S., Note on Kodaira-Spencer's proof of Lefschetz theorems, Proc. Jap. Acad., 30 (1954), 266-272.

[3] Andreotti, A. and Grauert, H., Théorème de finitude pour la cohomologie des espaces complexes, Bull. Soc. Math. France, 90 (1962), 193-259.

[4] Andreotti, A. and Vesentini, E., Carleman estimates for the Laplace-Beltrami equation on complex manifolds, Inst. Hautes Etudes Sci., Publ. Maih., 25 (1965), 81-130.

[5] Demailly, J. -P. and Skoda, H., Relations entre les notions de positivités de P. A. Griffiths et de S. Nakano pour les fibrés vectoriels, Seminaire Pierre Lelong-Henri Skoda, (Analyse) Années 1978/79, Lecture notes in mathematics, 822 (1980), 304-309.

[6] Fujiki, A., Hodge to de Rham spectral sequence on a strongly pseudoconvex manifold, preprint.

[ 7 ] Girbau, J., Sur le théorème de Le Potier d'annulation de la cohomologie, C. R. Acad. Sc. Paris, 283 (1976), 355-358.

[ 8 ] Griffiths, P. A., The extension problem in complex analysis II; embedding with positive normal bundle, Amer. J. of Math., 88 (1966), 366-446.

[9] - Hermitian differential geometry, Chern classes, and positive vector bundles, Global Analysis, papers in honor of K. Kodaira, edited by D. C. Spencer and S. Iyanaga, Univ. of Tokyo Press and Princeton Univ. Press 1969, 185-251.

[10] Hörmander, L., $L^{2}$ estimates and existence theorems for the $\bar{\partial}$ operator, Acta Math., 113 (1965), 89-152.

[11] - An Introduction to Complex Analysis in Several Variables, North-Holland Publishing Company, 1973.

[12] Kazama, H., Approximation theorem and application to Nakano's vanishing theorem for weakly 1-complete manifolds, Mem. Fac. Sci. Kyushu Univ., 27 (1973), 221-240.

[13] Kodaira, K., On Kähler varieties of restricted type, Ann. Math., 60 (1954), $28-48$.

[14] Leistner, D., Der Endlichkeitssatz mit Abschätzung für pseudokonvexe und pseudokonkave Räume, Dissertation Regensburg, 1974.

[15] Lieberman, D. and Rossi, H., Deformations of strongly pseudo-convex manifolds, Rencontre sur l'analyse complexe à plusieurs variables et les systèmes surdéterminés, les presses de l'université de Montréal, 1975, 119-165.

[16] Morrey, C. B., Multiple integrals in the calculus of variations, Springer, 1966.

[17] Nakano, S., On complex analytic vector bundles, J. M. S. Jap., 7 (1955), 1-12.

[18] - On the inverse of monoidal transformation, Publ. RIMS, Kyoto Univ., $6(1970 / 71), 483-502$.

[19] — Vanishing theorems for weakly 1-complete manifolds, Number Theory, Algebraic Geometry and Commutative Algebra, in honor of Y. Akizuki, Kinokuniya, Tokyo, 1973, 169-179.

[20] - On weakly 1-complete manifolds, Manifolds-Tokyo (Proceedings of the international conference on manifolds and related topics in topology), 1973, 323-327.

[21] — Vanishing theorems for weakly 1-complete manifolds, II, Publ. RIMS, Kyoto Univ., 10 (1974), 101-110. 
[22] Nakano, S. and Rhai, T. S., Vector bundle version of Ohsawa's finiteness theorems, Math. Japonica, 24 (1980), 657-664.

[23] Ohsawa, T., Finiteness theorems on weakly 1-complete manifolds, Publ. RIMS, Kyoto Univ., 15 (1979), 853-870.

[24] - On $H^{p, q}(X, B)$ of weakly 1-complete manifolds, Publ. RIMS, Kyoto Univ., 17 (1981), 113-126.

[25] —, A reduction theorem for cohomology groups of very strongly $q$-convex Kähler manifolds, Invent. Math., 63 (1981), 335-354.

[26] Skoda, H., Remarques a propos des théorèmes d'annulation pour les fibrés semipositifs, Seminaire Pierre Lelong-Henri Skoda (Analyse) 1978/79, Lecture Notes in Math., 822 (1980), 252-257.

[27] Suzuki, O., Simple proofs of Nakano's vanishing theorem and Kazama's approximation theorem for weakly 1-complete manifolds, Publ. RIMS, Kyoto Univ., 11 (1975), 201-211.

[28] Takeogoshi, K., A generalization of vanishing theorems for weakly 1-complete manifolds, Publ. RIMS, Kyoto Univ., 17 (1981), 311-330.

[29] Takegoshi, K. and Ohsawa, T., A vanishing theorem for $H^{p}\left(X, \Omega^{q}(B)\right)$ on weakly 1-complete manifolds, Publ. RIMS, Kyoto Univ., 17 (1981), 723-733.

[30] Vesentini, E., Lectures on Levi convexity of complex manifolds and cohomology vanishing theorems, Institute of Fundamental Research, Bombay, 1967.

[31] Weil, A., Introduction à l'etude des variétés kähleriennes, Act. Sci. Ind. 1267, Hermann, 1958.

[32] Wells, R. O., Differential analysis on complex manifolds, Prentice-Hall, Englewood Cliffs, N. J., 1973.

Added in proof: $\mathrm{O}$. Abdelkader has proved in "Un théorème d'approximation pour les formes à valeurs dans un fibré semi-positif, C. R. Acad. Sci. 293 (1981), 513-515" the result $H^{t}\left(X, \Omega^{s}(E)\right)=0$ in our Theorem 2.7 by a different method, but still using the approximation argument as in [12]. 